The Astrophysical Journal, 668:571-585, 2007 October 10

(C) 2007. The American Astronomical Society. All rights reserved. Printed in U.S.A.

\title{
DETERMINING THE SOURCE OF CORONAL HELICITY THROUGH MEASUREMENTS OF BRAIDING AND SPIN HELICITY FLUXES IN ACTIVE REGIONS
}

\author{
D. W. LONGCOPE AND B. RAVindRA \\ Department of Physics, Montana State University, Bozeman, MT \\ AND \\ G. BARNES \\ Colorado Research Associates Division, NorthWest Research Associates, 3380 Mitchell Lane, Boulder, CO 80301 \\ Received 2007 March 3; accepted 2007 June 21
}

\begin{abstract}
Magnetic helicity has become a valuable tool for understanding the energetics and dynamics of coronal magnetic fields. Recently, long time sequences of magnetograms have been used to measure the flux of helicity into active region coronae. We demonstrate how this helicity flux can be usefully decomposed into contributions of differing origin, called "spin" helicity and "braiding" helicity. These contributions could be envisioned to come at the expense of twist and writhe helicity, respectively, of a subphotospheric flux tube anchored to the regions. In order to effect this decomposition, each magnetogram is partitioned into a set of unipolar regions. We present a method of defining such regions so that they persist through the sequences and track the photospheric flow. The spin helicity of a given region quantifies the mean rotation rate of motions internal to that region, while braiding helicity is injected by the motions of whole regions about one another. Applying the method to six active regions shows cases where either spin or braiding dominates, and where they have the same signs and opposite signs. Thus, it would seem that no general statement can be made regarding the dominance of twist or writhe in supplying helicity to the corona. In one particular case, spin and braiding helicity follow different time histories but inject equal and opposite net helicities. This suggests that the spinning and braiding are driven by a kink instability in the submerged flux tube.
\end{abstract}

Subject headings: MHD — Sun: magnetic fields

\section{INTRODUCTION}

Magnetic helicity has emerged, over the past several years, as an important quantitative tool for characterizing the solar coronal field. Defined originally by Berger \& Field (1984), relative helicity quantifies the overall twist of a magnetic field anchored to a boundary such as the photosphere. Since relative helicity is typically defined relative to the field's lowest energy state, a potential field, it may be used to bound the system's "free energy," the energy above the minimum (Berger 1988). Unfortunately, relative helicity is not directly measurable since it depends on the magnetic field throughout the entire coronal volume. It is, however, conserved under conditions expected in the corona and therefore changes only from fluxes through boundaries (Berger 1984). This flux depends only on properties at the boundaries, such as the photosphere, and is therefore far more accessible to direct measurement.

The photospheric flux of relative helicity depends on the distribution of magnetic field and velocity there. Since magnetic field maps, "magnetograms," are standard measurements by now, the principal hurdle in any relative helicity flux measurement is mapping the photospheric velocity. The most widely used technique for constructing such a map is the "local correlation tracking" (LCT) introduced by November \& Simon (1988). Chae (2001) combined LCT velocity maps with 40 hours of sequential magnetograms from the Michelson Doppler Imager (MDI; Scherrer et al. 1995) to produce a time history of the helicity flux into one particular active region (AR). Subsequent authors have refined the technique (Kusano et al. 2002; Démoulin \& Berger 2003). One form or another has been applied to extended observation of several other active regions (Chae et al. 2001; Green et al. 2002; Démoulin et al. 2002; Moon et al. 2002; Nindos et al. 2003), leading to a developing picture of the magnetic helicity budget of a "typical" active region.

The aforementioned measurements confirm that magnetic helicity crosses the photosphere in most ARs observed. It is hypothesized that the additional free energy which this helicity represents is a driver of energetic activity such as flaring and coronal mass ejections. It is less clear what serves as the source driving helicity across the photosphere in the first place.

Since helicity is conserved, any change in the coronal helicity must be compensated by an offsetting change in the convection zone. The confinement of subphotospheric magnetic field to isolated flux permits its helicity to be decomposed into "twist" and "writhe" contributions (Berger \& Field 1984; Moffatt \& Ricca 1992). The writhe helicity of a flux tube depends only on the configuration of its axis, while the twist contribution depends on the internal structure of the field about the axis. This kind of decomposition cannot be performed naturally on the coronal field, since it fills the volume. It is, however, meaningful to ask whether photospheric helicity flux comes at the expense of twist or writhe helicity of the underlying flux tubes. It is this question we wish to address in the present work.

The primary motivation for answering this question is that we intuitively associate a different mechanism with changes in writhe than with changes in twist. A subphotospheric flux tube's writhe is changed by a motion of its axis. Such motions couple strongly to the external, unmagnetized plasma through aerodynamic drag forces, even at extremely high Reynold's numbers (Parker 1975; Choudhuri \& Gilman 1987). Since the magnetic energy density of the flux tube is small compared to the kinetic energy density of its surroundings, we consider changes in writhe to be "driven" by external flows.

Twist changes, on the other hand, can occur through spinning motion internal to the flux tube, which couples to the external, 
subphotospheric medium only through viscosity. At high Reynold's numbers, such coupling is expected to be extremely weak. No pressure field, however strong, can produce a torque on the tube $(\nabla \times$ $\nabla p=0$ ), so spinning motion is believed to be driven by magnetic forces alone (Longcope \& Welsch 2000). Thus, the tube plays an active role in producing its own twist changes, and a passive role in writhe changes - the latter result from external flows.

Photospheric flux concentrations, such as sunspots or active region polarities, are believed to be the ends of these subphotospheric flux tubes. Provided all photospheric motions are horizontal, then the above associations of internal motions with changes in twist and external motions with changes in writhe will apply to motions within the regions as well. Vertical flow might allow external motions to couple to twist. Berger \& Prior (2006) provide a rigorous formalism whereby the writhe of subphotospheric flux tubes can be calculated, even though they end at photospheric points. It is in principle possible to use this in a rigorous derivation of a relationship between changes in this writhe and the (external) motions of these footpoints. Such a derivation would, however, substantially divert our development. We will, instead, leave as intuitive and possibly inexact, the associations between twist and internal motions and between writhe and external motions

An example of a helicity change driven by external photospheric motions of regions is their passive advection by differential rotation. Several investigations have shown that this motion produces helicity fluxes far smaller than those observed in active regions (DeVore 2000; Démoulin et al. 2002). Significant helicity flux must therefore be attributable either to relative proper motions of photospheric flux elements about one another or to internal spinning motions within each element. Observations of the former have been used to infer the axis configurations of the underlying tube (Tanaka 1991; van Driel-Gesztelyi et al. 1994; Leka et al. 1996; Pevtsov \& Longcope 1998; López Fuentes et al. 2000). While not expressly calculated by these authors, helicity injection of this sort probably did occur in the cases they investigated. It is approximately correct to associate the integrated helicity flux of this kind with the emergence of the writhed portion of the flux tube through the photosphere. The exact relation can, however, be found using the formalism of Berger \& Prior (2006).

The alternative helicity source is magnetically driven, internal spinning of the flux tube itself. Longcope \& Welsch (2000) used a simplified model to show how an emerging twisted flux tube might develop such spinning footpoint motions. Driving the spinning is the torque caused by an unbalanced helicity density across the photospheric layer. The imbalance arises naturally as the AR expands into the corona, progressively diluting its helicity. Even as mass stays behind, horizontal spinning motion sends helicity into the corona. Pevtsov et al. (2003) measured coronal twist in six emerging ARs; in five of them the observed time evolution matched the Longcope \& Welsch (2000) curve given appropriate choices of parameters.

The most obvious cases where internal spinning motion is directly observed are the so-called rotating sunspots. ${ }^{1}$ Spinning motions were noted by numerous authors over the years (Evershed [1910] and St. John [1913] are among the earliest). Recently Brown et al. (2003, hereafter BN03) used high time cadence TRACE white light observations of seven different sunspots to measure spin rates. Using a stack-plot technique they obtain continuous measurements over many days at different radii from the umbra. Each of the seven spots exhibits rotation with a qualitatively different

\footnotetext{
1 For consistency with the foregoing discussion, we would prefer to call these "spinning" sunspots, to emphasize the internal nature of their motion. The term "rotating" has, however, already gained broad usage.
}

time history. In one case (AR 8668), the spot rotated steadily at $\sim 0.5^{\circ} \mathrm{hr}^{-1}$ for 5 days; in another (AR 9114), 30 hours of steady rotation $\left(\sim 1^{\circ} \mathrm{hr}^{-1}\right)$ was followed by a $30 \mathrm{hr}$ decline to zero; in yet another (AR 9077), the rotation rate fluctuated wildly, even changing direction. The one spot from a recently emerged region (AR 9004) showed a steady slowdown over 2.5 days, akin to the prediction of Longcope \& Welsch (2000).

Since both external and internal motions have been observed, it remains only to quantify their contribution to helicity flux into the entire AR. To do so we must be careful to account for spinning in all the flux of an AR, rather than just its largest sunspot. Moreover, the relative motions of these features will contribute helicity flux, but of the writhe-associated variety. If the spinning contribution is found to dominate the helicity flux in most ARs, then it is natural to think of coronal helicity as originating in the twist of the emerging flux tubes. Otherwise, it would seem that convection zone fluid motions play the leading role in creating the helicity which finds its way into the corona.

To obtain this insight into the source of helicity, we seek an observational means of distinguishing between twist-associated and writhe-associated helicity fluxes. A decomposition of this type was proposed by Berger (1984), when he considered the rigid, horizontal motions of isolated flux patches. (Berger [1991] and Welsch \& Longcope [2003] treat slightly more general flows.) The "braiding " and "spinning" terms of this decomposition yield the helicity flux contributed by motions external and internal to the patch, respectively. As straightforward as the decomposition is, its only observational use was by Welsch \& Longcope (2003), in finding the braiding helicity flux in the quiet Sun. The principal obstacle to its broader application, say to ARs, comes from the difficulty in distinguishing internal from external motion in a photospheric field not composed of truly isolated flux patches.

An observational helicity flux decomposition would shed light into the subphotospheric helicity source and would also guide modeling of coronal energy storage. Energy released in flares or coronal mass ejections seems to be converted from free magnetic energy built up from prior evolution of the photospheric flux distribution. As it becomes possible to simulate this process in complex three-dimensional geometries it is necessary to know which aspect of the photospheric evolution plays the principal role in storing the free energy. Some coronal field models (e.g., Longcope 1996) focus on relative motions of flux elements (i.e., braiding) while others focus on the spinning of the elements.

The present work generalizes the Berger (1984) decomposition to be applicable to arbitrary flux distributions and arbitrary flow fields. The decomposition hinges on a "partitioning" of the photospheric field into distinct unipolar regions. We present an algorithm for performing this partitioning on an extended time sequence of magnetograms, such as those produced by MDI. This partition defines what is meant by internal and external motions, and thus how spinning is distinguished from braiding. While the task is not well defined, our algorithm appears to produce a result satisfying physical expectations of such a distinction, including preservation under evolution of the photospheric flux.

Methods have been recently proposed for decomposing relative helicity into "self" and "mutual" contributions (Régnier et al. 2005; Démoulin et al. 2006; Low 2006). These are decompositions of the entire helicity integral, usually based on a partition of the "coronal volume" into distinct flux tubes (Berger \& Field 1984). The self-helicity of one tube characterizes the internal winding of field lines within it, while the mutual helicity of the collection of tubes characterizes their winding about one another. This decomposition concerns the instantaneous configuration of the entire volume rather than motions at the boundary. As such, it is 
fundamentally different from our decomposition of the helicity flux into "spin" and "braiding" based on a partition of the boundary field. While the spin helicity flux is sometimes assumed to be the source of self-helicity, there is no mathematical basis for such an association. Properly relating boundary motions to self-helicity or mutual helicity requires knowledge of how the coronal field interconnects the different photospheric flux patches (Pariat et al. 2005). Our decomposition of helicity flux requires no such knowledge and therefore does not discriminate self from mutual helicity sources.

Using our method we calculate the spin helicity and braiding helicity fluxes in several ARs. We find that the most accurate measurement of the spin contribution requires the highest possible spatial resolution for both the magnetogram and the velocity map. Using lower resolution can result in an underestimate of the internal spinning contribution, partly due to the greater smoothing of the velocity field from larger apodizing windows, but mostly due to systematically lower velocity values. This may have affected previous calculations of total helicity flux, since that includes both spin and braiding.

When we analyze ARs where sunspot rotation has been previously measured we find a spin helicity flux proportional to the rotation rate. In some cases this spin contribution dominates, suggesting helicity originating as a twisted flux tube. In other cases, even in the presence of rotating sunspots, braiding is the dominant contribution to helicity flux. Moreover, there are cases where the two contributions are of the same sign and cases where they oppose one another. In one particular case (AR 9004), we find evidence that the spinning and braiding is driven by a kink mode. It therefore appears that no general statement may be made to the origin of helicity in all cases. Nor is a single variety of photospheric stressing responsible for energy storage in all cases. The method presented here does, however, answer these questions in each specific case.

The paper is structured as follows. We demonstrate, in $\S 2$, our generalized decomposition using an analytic test case with known helicity flux. In order to apply the decomposition to a sequence of actual magnetograms it is necessary to automatically identify and track the region boundaries used to distinguish internal from external motions. We describe our algorithm in $\S 3$, and demonstrate that it does a reasonable job preserving moments of the region fluxes. In $\S 4$ we test the algorithm by comparing its spin rates to those of several of the sunspots measured by BN03. Comparisons reveal that accurate determination of internal flows requires high spatial and temporal resolution; lower resolution does reveal the spinning motion, but underestimates its magnitude. We then consider the compositions of the helicity fluxes for several whole ARs in $\S 5$. These cases include a broad range of different behaviors, including a case apparently driven by external kink mode. Finally, we summarize our method and its application in $\S 6$.

\section{THE GENERAL METHOD}

The decomposition of helicity flux requires that the vertical magnetic field of the photosphere, $B_{z}(x, y, 0)$, be "partitioned" into a set of unipolar regions. We begin by assuming this has been done and return in $\S 3$ to demonstrate how it is done in practice. A particular region, $\mathcal{R}_{a}$, is enclosed by a simple closed boundary $\partial \mathcal{R}_{a}$. The field within a given region may be characterized by its net flux and centroid location

$$
\begin{aligned}
\Phi_{a} & =\int_{\mathcal{R}_{a}} B_{z}(x, y, 0) d^{2} x, \\
\bar{x}_{i}^{a} & =\frac{1}{\Phi_{a}} \int_{\mathcal{R}_{a}} x_{i} B_{z}(x, y, 0) d^{2} x,
\end{aligned}
$$

where $i=1$ and 2 label the horizontal vector component. The next moment describing the flux distribution is the second rank tensor

$$
Q_{i j}^{a}=\frac{1}{\Phi_{a}} \int_{\mathcal{R}_{a}}\left(x_{i}-\bar{x}_{i}^{a}\right)\left(x_{j}-\bar{x}_{j}^{a}\right) B_{z}(x, y, 0) d^{2} x,
$$

related to the traditional quadrupole moment. A simple measure of a region's horizontal extent is its radius of gyration

$$
\bar{r}_{a} \equiv \sqrt{\operatorname{Tr}\left(\boldsymbol{Q}^{a}\right)}
$$

We assume that all significant flux is assigned to one of the regions and assume $B_{z}=0$ everywhere outside our set of regions.

The partitioning turns out to be critical, since it determines how the spin and braiding helicities are defined. In cases where unipolar regions are clearly separated by regions of very small field strength, it is straightforward and unambiguous. We return below to discuss the more complex cases, but illustrate our method in a simple case: a quadrupolar configuration shown in Figure 1, similar to that considered by Longcope \& Magara (2004). The flux is clearly separated into four regions which are numbered 1-4 (the $P$ or $N$ indicate polarity). Two of the regions, $\mathrm{P} 1$ and N2, are initially circular, while the other two are elliptical. All four have the same magnitude of flux; the centroids are indicated by plus signs and crosses.

We next consider a horizontal, photospheric velocity field $\boldsymbol{u}(x, y)$. The flux-weighted velocity within region $a$ is given by

$$
\bar{u}_{i}^{a} \equiv \frac{1}{\Phi_{a}} \int_{\mathcal{R}_{a}} u_{i}(x, y) B_{z}(x, y, 0) d^{2} x .
$$

If the vertical field and the regions are both advected by this flow then it is possible to show that $d \Phi_{a} / d t=0$ and that the change in centroid position is given by

$$
\frac{d \overline{\boldsymbol{x}}^{a}}{d t}=\overline{\boldsymbol{u}}^{a} .
$$

If there is some vertical flow $v_{z}$ across the photosphere in addition to a horizontal component $v_{h}$, these can be combined into a horizontal "pattern velocity,"

$$
\boldsymbol{u}=\boldsymbol{v}_{h}-\frac{v_{z}}{B_{z}} \boldsymbol{B}_{h},
$$

which governs the advection of $B_{z}$ as long as the full ideal induction equation is obeyed in the photospheric plane (Démoulin $\&$ Berger 2003). If this pattern velocity is everywhere defined (i.e., there is no vertical flow at polarity inversion lines) and region boundaries are also advected by $\boldsymbol{u}$, then $\dot{\Phi}_{a}=0$ and equation (6) still obtains. We will henceforth assume this to be the case, and therefore neglect possible effects of flux emergence or submergence.

We choose to evolve our example field using a simple horizontal flow field restricted to the central disk outlined by the dotted circle in Figure 1. We take the flow to be that of a point vortex at the origin, $v_{\phi}=C / r$, where the constant $C$, is chosen to give the rotation rate unity at the radius where $\mathrm{P} 1$ and $\mathrm{N} 2$ are initially centered. The motion is then parameterized by the angle $\psi$ by which this radius is rotated. This strongly sheared flow distorts the central regions as shown by the right panel of Figure 1. This distortion causes the centroids to move inward and the radius of gyration to increase. By the later stages (such as the right panel of Fig. 1) the two regions become intertwined. 

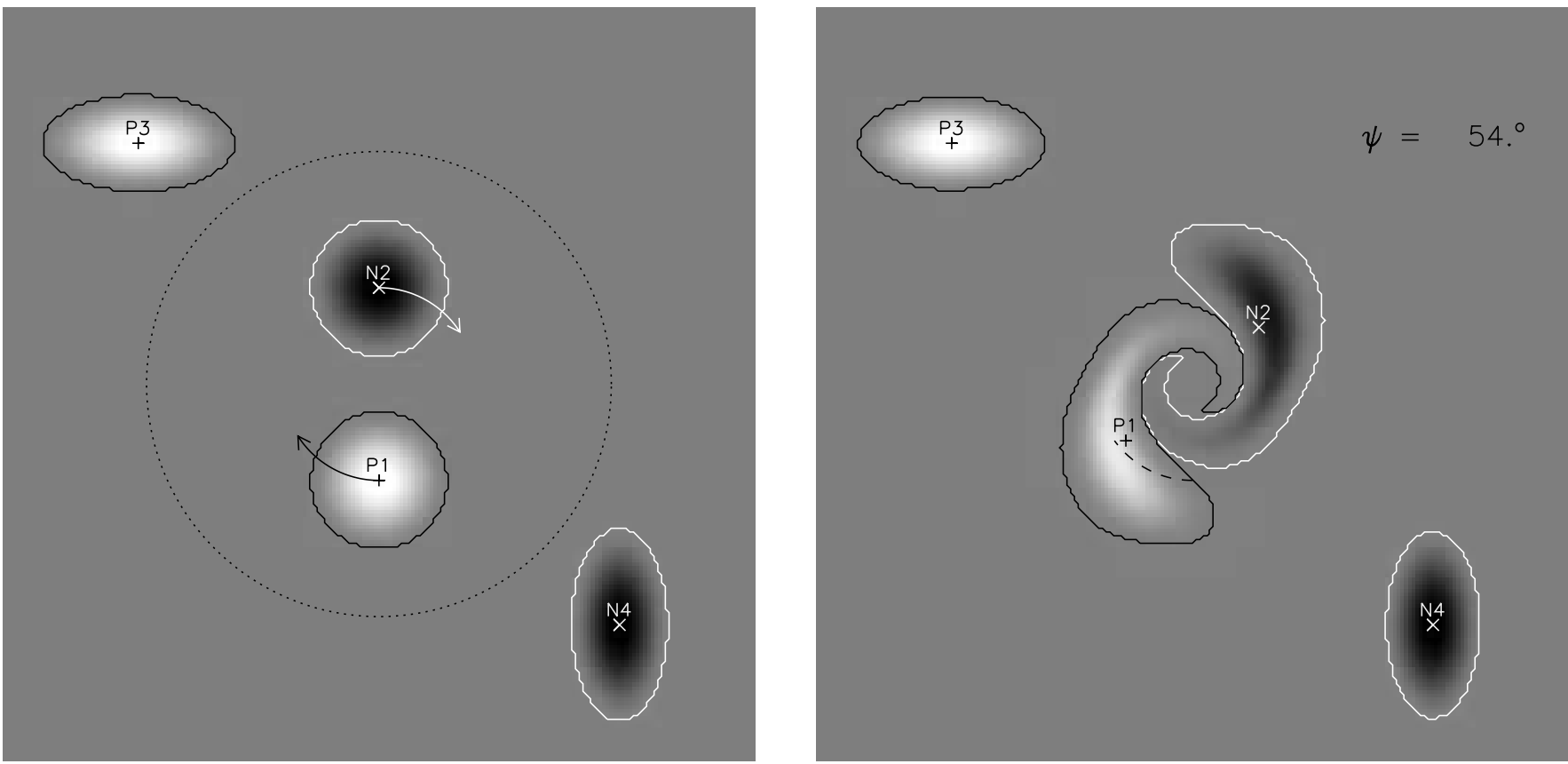

FIG. 1.-Quadrupolar configuration used to illustrate the helicity flux decomposition. Left: Initial configuration with two circular regions, P1 and N2 and two elliptical regions, $\mathrm{P} 3$ and N4. The central portion, inside the dotted line, undergoes a clockwise rotation, causing P1 and N2 to rotate about the center (indicated by arrows). Right: State after application of a motion, $v_{\phi}=C / r$, over an interval of time. This flow moves the point corresponding initially to the centroid of $\mathrm{P} 1$ along the dashed line over an angle $\psi=54^{\circ}$. Distortion of the region has caused its centroid to move inward slightly.

\subsection{Helicity Fluxes}

The relative helicity of the coronal magnetic field is defined by the volume integral

$$
H=\int_{z>0}\left(\boldsymbol{A}+\boldsymbol{A}_{P}\right) \cdot\left(\boldsymbol{B}-\boldsymbol{B}_{P}\right) d^{3} x
$$

where $\boldsymbol{B}_{P}$ is a potential magnetic field $\left(\nabla \times \boldsymbol{B}_{P}=0\right)$ matching the photospheric vertical field and $\boldsymbol{A}$ and $\boldsymbol{A}_{P}$ are vector potentials for the actual field and the potential field, respectively. If the coronal field evolves without resistivity, or other nonideal electric fields, then its helicity changes only due to photospheric motions according to

$$
\dot{H}=-2 \int_{z=0}\left(\boldsymbol{u} \cdot \boldsymbol{A}_{P}\right) B_{z} d^{2} x,
$$

where $\boldsymbol{u}(x, t)$ combines the horizontal and vertical plasma velocities according to expression (7).

The horizontal components of $\boldsymbol{A}_{P}(\boldsymbol{x})$ at the photosphere $(z=0)$ are directly related to the vertical magnetic field through a Green's function:

$$
\boldsymbol{A}_{P}(\boldsymbol{x})=\frac{1}{2 \pi} \int_{z^{\prime}=0} \frac{\hat{z} \times\left(\boldsymbol{x}-\boldsymbol{x}^{\prime}\right)}{\left|\boldsymbol{x}-\boldsymbol{x}^{\prime}\right|^{2}} B_{z}\left(\boldsymbol{x}^{\prime}\right) d^{2} x^{\prime} .
$$

We can decompose the full vector potential into a sum of contributions from each region by restricting the region of integration:

$$
\boldsymbol{A}_{P}^{b}(\boldsymbol{x})=\frac{1}{2 \pi} \int_{\mathcal{R}_{b}} \frac{\hat{z} \times\left(\boldsymbol{x}-\boldsymbol{x}^{\prime}\right)}{\left|\boldsymbol{x}-\boldsymbol{x}^{\prime}\right|^{2}} B_{z}\left(\boldsymbol{x}^{\prime}\right) d^{2} x^{\prime} .
$$

This is valid for $\boldsymbol{x}$ inside and outside $\mathcal{R}_{b}$. The full vector potential is then $\boldsymbol{A}_{P}=\sum_{b} \boldsymbol{A}_{P}^{b}$.
Using these components the helicity flux can then be decomposed into a sum of two terms:

$$
\begin{aligned}
\dot{H}= & -2 \sum_{a} \int_{\mathcal{R}_{a}}\left[\boldsymbol{A}_{P}^{a}(\boldsymbol{x}) \cdot \boldsymbol{u}(\boldsymbol{x})\right] B_{z}(\boldsymbol{x}) d^{2} x \\
- & \frac{1}{\pi} \sum_{a} \sum_{b \neq a} \int_{\mathcal{R}_{a}} \int_{\mathcal{R}_{b}}\left[\frac{\hat{z} \times\left(\boldsymbol{x}-\boldsymbol{x}^{\prime}\right) \cdot \boldsymbol{u}(\boldsymbol{x})}{\left|\boldsymbol{x}-\boldsymbol{x}^{\prime}\right|^{2}}\right. \\
& \left.\times B_{z}\left(\boldsymbol{x}^{\prime}\right) B_{z}(\boldsymbol{x}) d^{2} x^{\prime} d^{2} x\right],
\end{aligned}
$$

which are the spin and braiding term, respectively (Welsch \& Longcope 2003). The first term is a sum of spinning helicity from each of the regions independently. Each term can be written using an average rotation rate, $\bar{\omega}_{a}$,

$$
\dot{H}_{\mathrm{sp}}^{a}=-2 \int_{\mathcal{R}_{a}}\left[\boldsymbol{A}_{P}^{a}(\boldsymbol{x}) \cdot \boldsymbol{u}(\boldsymbol{x})\right] B_{z}(\boldsymbol{x}) d^{2} x \equiv-\frac{\Phi_{a}^{2} \bar{\omega}_{a}}{2 \pi} .
$$

This expression is gauge-invariant and well defined, since it corresponds to the total helicity which would be injected if $\mathcal{R}_{a}$ were alone on the surface. If the motion within $\mathcal{R}_{a}$ is combination of translation and rigid rotation then $\bar{\omega}_{a}$ will be the rotation rate, regardless of how the flux is distributed (Berger 1984). For a general flow field, however, expression (13) defines a mean angular rotation $\bar{\omega}_{a}$ for the region,

$$
\bar{\omega}_{a} \equiv \frac{4 \pi}{\Phi_{a}^{2}} \int_{\mathcal{R}_{a}}\left[\boldsymbol{A}_{P}^{a} \cdot\left(\boldsymbol{u}-\overline{\boldsymbol{u}}_{a}\right)\right] B_{z} d^{2} x
$$

where the mean velocity has been removed using the fact that a constant velocity field produces zero in expression (13). While we call this the "mean" rotation, expression (14) does not coincide with a straightforward averaging process. 

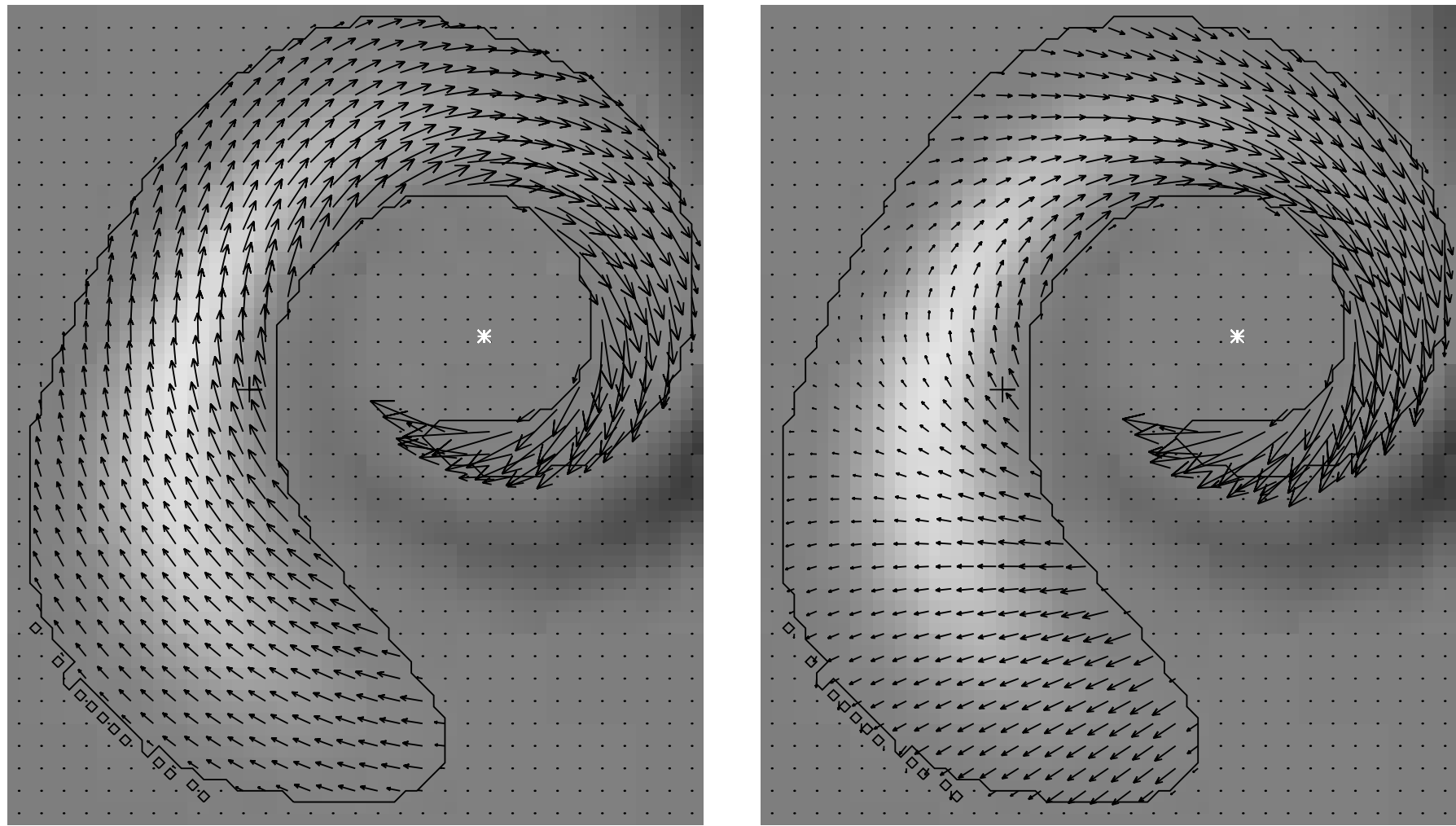

FIG. 2.-Velocity field within region P1 at $\psi=90^{\circ}$ Left: Arrows showing the field $\boldsymbol{u}(x, y)$ within the region. The gray scale is $B_{z}(x, y)$, and the solid curve outlines $\mathcal{R}_{1}$. A plus sign is plotted at the centroid and an asterisk at the origin, about which the flow circulates. Right: Same as the left panel, but for the field with the mean velocity removed: $\boldsymbol{u}-\overline{\boldsymbol{u}}_{1}$.

Figure 2 illustrates the procedure through its application to region $\mathrm{P} 1$ at the end of its evolution $\left(\psi=90^{\circ}\right)$. The left panel shows the flow field within the region $\mathcal{R}_{1}$. Integrating this over the region as equation (5) gives the mean region velocity, $\overline{\boldsymbol{u}}_{1}$, which is mostly azimuthal (clockwise) but contains an inward radial component. Subtracting this from $\boldsymbol{u}(x, y)$ yields the flow field plotted on the right. Performing the integral in equation (14) gives the value $\bar{\omega}_{1}=-0.63$ at this time. Performing these steps over the entire sequence yields the curves shown in Figure 3 . The spin rate $\bar{\omega}_{1}$ (solid curve) begins very small, perhaps because the flow field itself is irrotational except at the origin. Over time it become increasingly negative until it reaches the value $\bar{\omega}_{1}=-0.63$ of Figure 2 . The azimuthal rotation rate is initially $\bar{u}_{\phi} / r=-1$, chosen in the definition of $\boldsymbol{u}(x, y)$. As the region wraps about the origin,

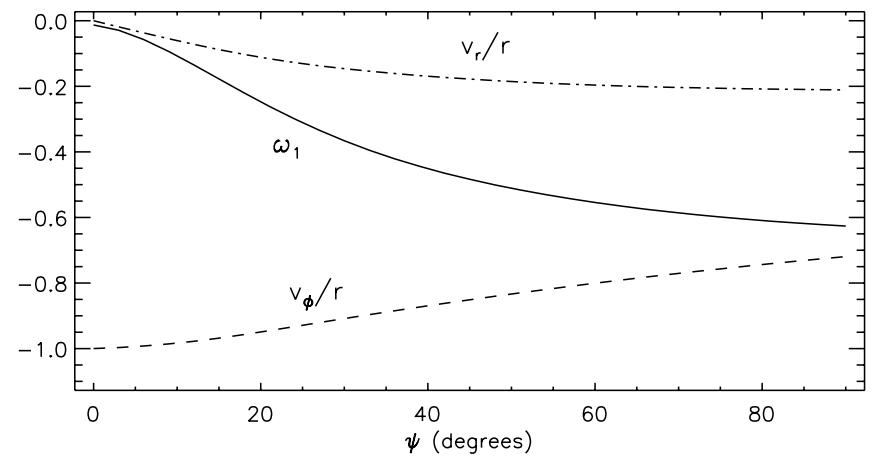

FIG. 3.-Various frequencies from the moments of region P1 as the magnetic field evolves. The functions are plotted against the rotation angle $\psi$. The solid curve is the mean spin rate, $\bar{\omega}_{1}$, describing its helicity injection. The dashed and dashdotted curves are the azimuthal rotation rate, $\bar{u}_{\phi} / r$, and radial expansion rate, $\bar{u}_{r} / r$, respectively. In the plot, these are labeled as " $v$ " rather than " $u$." however, its azimuthal rotation slows and the centroid approaches the origin.

The double sum in equation (12) is the exact braiding helicity for this partition:

$$
\begin{aligned}
\dot{H}_{\mathrm{br}}= & -\frac{1}{\pi} \sum_{a} \sum_{b \neq a} \int_{\mathcal{R}_{a}} \int_{\mathcal{R}_{b}}\left[\frac{\hat{z} \times\left(\boldsymbol{x}-\boldsymbol{x}^{\prime}\right) \cdot \boldsymbol{u}(\boldsymbol{x})}{\left|\boldsymbol{x}-\boldsymbol{x}^{\prime}\right|^{2}}\right. \\
& \left.\times B_{z}\left(\boldsymbol{x}^{\prime}\right) B_{z}(\boldsymbol{x}) d^{2} x^{\prime} d^{2} x\right] .
\end{aligned}
$$

The integrals can be expanded in powers of the separations between regions $\mathcal{R}_{a}$ and $\mathcal{R}_{b}$ to yield a simplified expression approximating the braiding helicity:

$$
\dot{H}_{\mathrm{br}} \simeq-\frac{1}{2 \pi} \sum_{a} \sum_{b \neq a} \Phi_{a} \Phi_{b} \frac{\hat{z} \times\left(\overline{\boldsymbol{x}}_{a}-\overline{\boldsymbol{x}}_{b}\right) \cdot\left(\overline{\boldsymbol{u}}_{a}-\overline{\boldsymbol{u}}_{b}\right)}{\left|\overline{\boldsymbol{x}}_{a}-\overline{\boldsymbol{x}}_{b}\right|^{2}} .
$$

This neglects terms which are smaller by factors typically $\sim \bar{r}_{b}^{2} / \mid \bar{x}_{a}-$ $\left.\overline{\boldsymbol{x}}_{b}\right|^{2}$ or $\sim \bar{r}_{a}^{2} /\left|\overline{\boldsymbol{x}}_{a}-\overline{\boldsymbol{x}}_{b}\right|^{2}$. It involves only the rates at which the different centroids braid about one another.

Figure 4 shows the various helicities calculated by integrating the time derivatives $\dot{H}$. Integrating the exact helicity flux, equation (9) gives the total helicity, plotted as a solid curve. Integrals of the spin term, equation (13), and the approximate braiding term, equation (16), are plotted as plus signs and crosses, respectively. The asterisks show the sum of these contributions, which approximates the total helicity. The discrepancy between the asterisks and the solid line is due to approximating the braiding helicity contribution by the motions of the region centroids. The exact braiding helicity, equation (15), is plotted as a dashed curve. Adding this to 


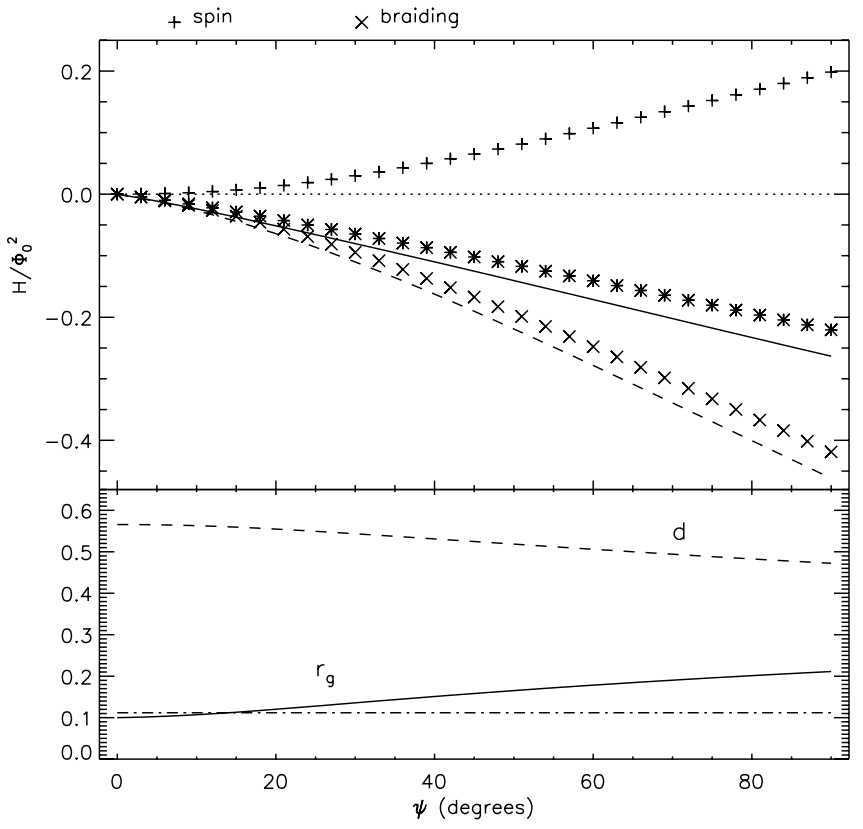

FIG. 4.-Helicity estimated for the vortical advection of the quadrupolar test case vs. the angle $\psi$ of rotation at the initial centroids. Top: Spin ( plus signs) and braiding (crosses) helicities and their sum (asterisks) as a function of $\psi$. The exact injected helicity, calculated using eq. (9), is shown by a solid line. The braiding helicity shown by crosses is calculated from eq. (16); this is only an approximation of the exact braiding helicity (dashed curve) from eq. (15). Bottom: Radii of gyration of the inner regions (solid curve) and outer regions (dash-dotted curve) as a function of $\psi$. The separation between the centroids of the inner regions is plotted as a dashed line.

the spin helicity ( plus signs), which is not approximated, will yield the solid curve exactly. The discrepancy between the dashed curve (exact) and the crosses (approximate) is due to the neglect of terms higher order in $\bar{r}_{a} /\left|\overline{\boldsymbol{x}}_{a}-\overline{\boldsymbol{x}}_{b}\right|$, involving the quadrupole moments. The growing significance of these contributions could be predicted by the convergence of $d$, the separation between $\mathrm{P} 1$ and $\mathrm{N} 2$ centroids, and their $\bar{r}$, in the bottom panel.

\subsection{Merging and Fragmentation}

The decomposition relies on a partitioning step which defines a particular set of regions. Using a different partitioning of the same field will result in a different decomposition. To illustrate the effect of different partitioning consider modifying an existing partition by merging two regions into one new one: $\mathcal{R}_{a}=\mathcal{R}_{a 1} \cup \mathcal{R}_{a 2}$. Straightforward application of equations (1) and (2) show that

$$
\Phi_{a}=\Phi_{a 1}+\Phi_{a 2}, \quad \overline{\boldsymbol{x}}_{a}=\frac{\Phi_{a 1}}{\Phi_{a}} \overline{\boldsymbol{x}}_{a 1}+\frac{\Phi_{a 2}}{\Phi_{a}} \overline{\boldsymbol{x}}_{a 2},
$$

as one might expect. The mean angular rotation of the merged region is

$$
\begin{aligned}
\bar{\omega}_{a} & =\frac{\Phi_{a 1}^{2}}{\Phi_{a}^{2}} \bar{\omega}_{a 1}+\frac{\Phi_{a 2}^{2}}{\Phi_{a}^{2}} \bar{\omega}_{a 2}+\frac{2 \Phi_{a 1} \Phi_{a 2}}{\Phi_{a}^{2}} \frac{d \theta_{12}}{d t}, \\
& =\frac{d \theta_{12}}{d t}+\frac{\Phi_{a 1}^{2}}{\Phi_{a}^{2}}\left(\bar{\omega}_{a 1}-\frac{d \theta_{12}}{d t}\right)+\frac{\Phi_{a 2}^{2}}{\Phi_{a}^{2}}\left(\bar{\omega}_{a 2}-\frac{d \theta_{12}}{d t}\right),
\end{aligned}
$$

where $\theta_{12}$ is the polar angle of the separation $\overline{\boldsymbol{x}}_{a 2}-\overline{\boldsymbol{x}}_{a 1}$.

According to equation (17), the spin helicity of $\mathcal{R}_{a}$ is the sum of the spin helicities of its components, $\mathcal{R}_{a 1}$ and $\mathcal{R}_{a 2}$ plus the part of the braid helicity involving those two about one another. The braiding helicity of the new partition is naturally decreased by the same amount,

$$
\Delta \dot{H}_{\mathrm{br}} \simeq-\frac{1}{\pi} \Phi_{a 1} \Phi_{a 2} \frac{d \theta_{12}}{d t} .
$$

Merging the two regions thereby converted the braiding helicity into spin helicity. From the final expression in equation (17) it is apparent that if all of region $a$ rotates rigidly (so $\bar{\omega}_{a 1}=\bar{\omega}_{a 2}=$ $d \theta_{12} / d t$ ), then the process of merging simply redefines the braiding of the two elements into the spin of the composite.

Conversely, fragmenting a region into two parts will transform some of the original spin helicity into braiding helicity. If fragmentation were applied recursively to the fragments - breaking pieces into ever smaller pieces - the total spin helicity contribution would vanish and the entire helicity flux would become braiding helicity. This is the conceptual limit in which every field line becomes its own flux tube and the total helicity quantifies their interlinking. The limit is illustrative, but not practical, since we wish to distinguish between helicity generation mechanisms operating internally and externally to a subphotospheric flux tube.

\section{MAGNETOGRAM PARTITIONING}

We apply the above algorithm to sequences of magnetograms from MDI (Scherrer et al. 1995) at either high resolution $\left(0.6^{\prime \prime} \times 0.6^{\prime \prime}\right.$ pixels, within a restricted field) or low resolution $\left(2^{\prime \prime} \times 2^{\prime \prime}\right.$ over the entire solar disk). In either case we begin with a data cube constructed by extracting rectangular sections enclosing the principal flux in the AR. Following Berger \& Lites (2003) the magnetic field strength in each pixel is multiplied by a calibration factor of 1.56. To reduce the noise in the magnetic field measurement, five successive 1 minute magnetograms are added, thereby reducing the noise to $\approx 9 \mathrm{G}$. Assuming the magnetic field is radial at the photosphere, the line-of-sight component is divided by the cosine of the angle from the disk center to convert to vertical field.

Local correlation tracking is performed on the sequence using an apodizing window of 7 " on the low-resolution and $4.5^{\prime \prime}$ on the high-resolution sequence (November \& Simon 1988). Correlation tracking is applied to pairs of magnetograms separated in time by $1 \mathrm{hr}$ for the low resolution and 20 minutes for the high resolution. Only pixels whose magnetic field strength is larger than $50 \mathrm{G}$ are correlated; this reduces the computing time and prevents noisy quiet Sun pixels from affecting the velocities. Parameters in the LCT steps were chosen after experimentation. It was found that larger apodizing windows were best used in conjunction with larger time steps. The size of the apodizing window, and therefore of the time step, was also dictated by pixel size.

The critical step in applying techniques outlined in $\S 2$ is to partition the active region field into distinct flux concentrations. To be useful in characterizing AR evolution it is necessary to track the evolution of these flux concentrations over a long time interval, preferably on the order of a few days. The approach we present here builds on a gradient-based tessellation scheme (Schrijver et al. 1997; Hagenaar 1999; Barnes et al. 2005). One partition consists of all pixels which are strictly downhill from a local maximum.

This scheme works well on any single magnetogram, but when applied to a time series, it can lead to large variations in the partitioning from one time step to the next. In particular, it is frequently the case that a partition assigned to a single maximum at one time will, at the next time, contain two local maxima splitting the partition into two. To mitigate effects such as this, we use an approach, similar to Barnes et al. (2005) of adjusting each partition to minimize its differences with a reference. Barnes et al. (2005) constructed a 
reference by averaging an approximately hour-long time series of magnetograms with a cadence of a few minutes. In the present case, that approach will no longer work because we are interested in time series lasting longer than a day, with cadences of hours rather than minutes.

Instead of a time-averaged reference for the entire time series, we construct a new reference for each magnetogram by advecting, with the LCT velocity field, the partition from a neighboring time step. For examples presented here, which contain regions of rapid flux emergence, we find it preferable to start with the last time step and advect the partitions backward in time. Instances where a new source region appears will seem, in the reversed time, to represent the disappearance of an existing, labeled partition.

For the initial time we first smooth the field by performing a potential field extrapolation to a height of 1 pixel. This reduces the number of local maxima in $\left|B_{\text {los }}\right|$ resulting from noise. The gradient-based tessellation scheme is then applied to assign a label to each pixel with a field strength exceeding $50 \mathrm{G}$.

This results in such a large number of partitions (typically several hundred) in regions of plage as to be unwieldy for subsequent calculations. To simplify the plage while maintaining the structure in sunspots, we apply a saddle point merging. That is, we evaluate the field at the saddle point between adjacent partitions, and if it is within $300 \mathrm{G}$ of either maxima of the two partitions, the smaller flux partition is assigned the same label as the larger flux partition. With this criterion, we are able to simplify the plage until a tractable number of sources is obtained (generally less than 100). Our hope in this approach is that the majority of the helicity injected into the corona comes from regions of strong fields, so the exact representation of weak fields is not crucial.

The prototypical case studied by BN03 was the leading sunspot in AR 9114, which they followed over 2000 August 8-10. This same region turns out to be a good test bed for our method since sequences of MDI magnetograms exist at both of the instruments' resolutions. We use a low-resolution sequence running from 16:24 on 2000 August 7 to 22:03 on August 11 and a high-resolution sequence covering the period from 15:12 UT on 2000 August 8 to 1:53 UT on August 9.

Figure 5 shows partitions from approximately the same time in each sequence. Although the regions and the naming do not coincide between the different data sets, in each one P01 designates the main leading flux concentration which includes the sunspot measured by BN03. Note that the region P01 in the lowresolution sequence encompasses not just the region $\mathrm{P} 01$ in the high-resolution sequence, but also several other small partitions. The low-resolution sequence includes flux that is outside the field of view for the high-resolution sequence.

The partition for the low-resolution case is summarized by the two histograms in Figure 6. The histogram of total flux, on the left, shows that $\sim 85 \%$ of the flux in each polarity falls above the threshold field strength of $50 \mathrm{G}$. All but a very small portion of this is assigned to partitions; the little not included belonged to regions deemed too small to matter. It is clear from the histogram that there is more total flux of the leading (positive) polarity within our window, and that it is systematically stronger.

The histogram on the right of Figure 6 shows the flux assigned to each of the different partitions at the time being considered. The largest by far is $\mathrm{P} 01$, by itself containing more than half of all the positive polarity in the $\mathrm{AR}\left(1.1 \times 10^{22} \mathrm{Mx}\right)$. This region contains a large sunspot where MDI magnetic field measurements are known to be less sensitive (Berger \& Lites 2003). In the present case there is $3.0 \times 10^{21} \mathrm{Mx}$, above $B=1700 \mathrm{G}$, all of it positive. Integrating only the amount by which the reported field exceeds this value yields $5.8 \times 10^{20} \mathrm{Mx}$. Since weak flux in P01
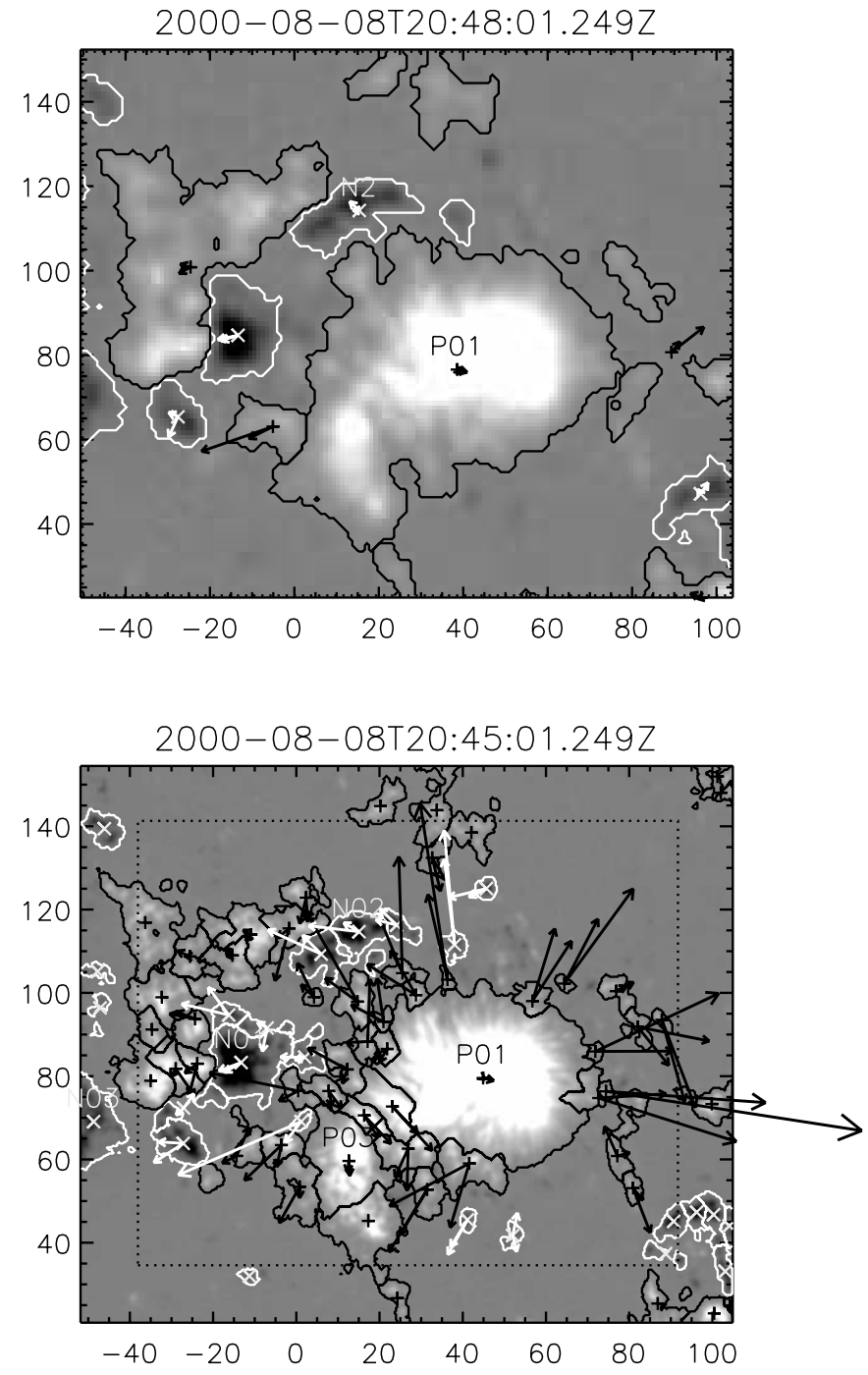

FIG. 5.-Partitioning of AR 9114 for a low-resolution magnetogram (top) and a high-resolution magnetogram (bottom), taken at approximately the same time. In both cases, axes are labeled in arcseconds from disk center. The low-resolution image covers a larger field of view. The dotted line bounds the region in which the LCT velocity is determined. For partitions entirely within this region, the velocities as determined both by LCT and by fitting the location of the centroid are shown as arrows, with length proportional to the speed. There is qualitatively good agreement in the magnitude and direction for both cases, although note that the speeds in the low-resolution case tend to be smaller than in the high-resolution case. The region labeled P01 contains the sunspot considered by BN03.

constitutes such a large fraction of its flux, our calculation of its total flux is probably only slightly compromised by insensitivity to strong field. The integrated field excess is about $5 \%$ of the total flux, so we expect any sensitivity-related error to be of similar size (Longcope et al. 2005).

To be applicable to helicity calculation, the partitions must follow the photospheric field. To ascertain how faithfully they do, we test the consequence of the tracking with equation (6); the centroid velocity of a partition, $d \overline{\boldsymbol{x}}_{a} / d t$, will match the flux-weighted average velocity within the partition, $\overline{\boldsymbol{u}}_{a}$.

To accurately determine the centroid velocity, we fit the centroid positions with a constant velocity over several successive time steps. Doing so for our partition of AR 9114 shows a qualitative tendency for the velocities to be of similar magnitude and in a similar direction, as shown by the arrows in Figure 5. The scatter plot shown in Figure 7 provides a more quantitative comparison. The slope of the regression line is close to unity for data 

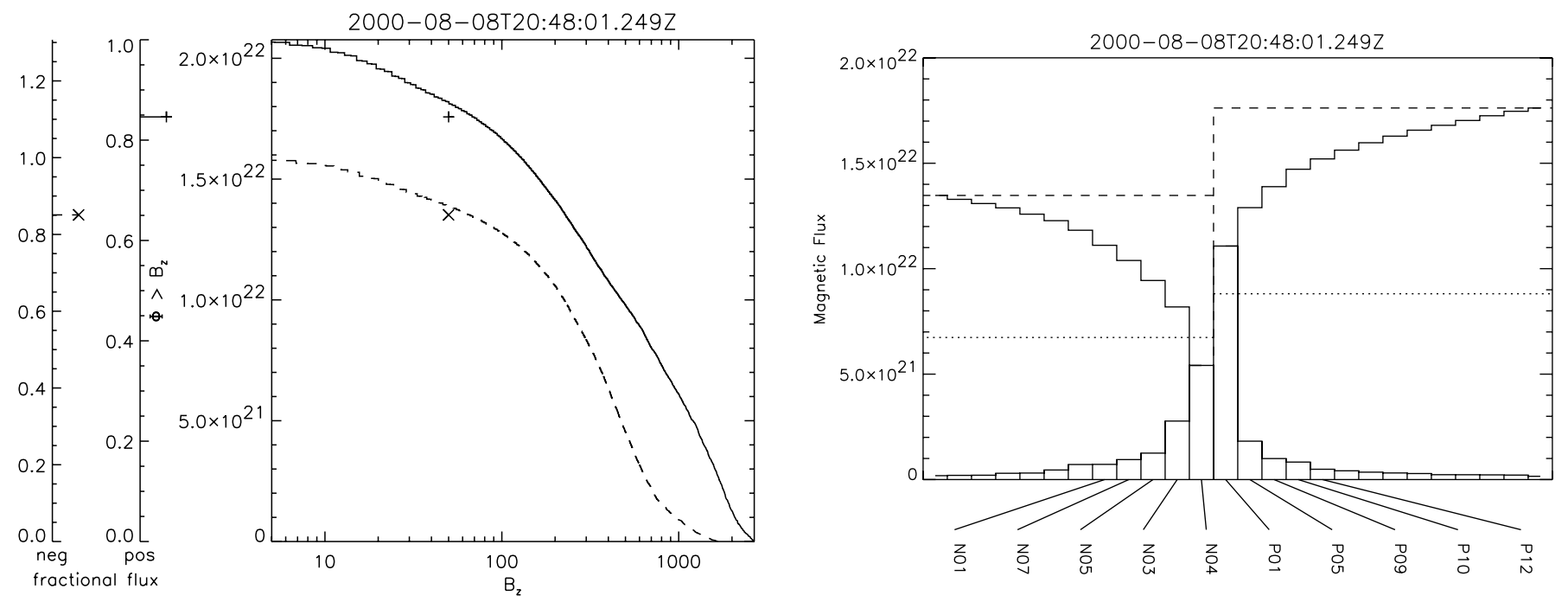

FIG. 6.-Histograms summarizing the partitioning of the low-resolution magnetogram from Fig. 5. Left: Cumulative histogram of the positive (negative) flux is shown as a solid (dashed) curve. The threshold level of $B=50 \mathrm{G}$ is indicated by a plus sign (cross). Their vertical placement indicates the amount included in the partitions, after discarding small regions. Right: Amount of flux in each individual region indicated by vertical bars decreasing rightward (leftward) from the center for positive (negative) elements. The cumulative flux is indicated by the stair-step curve above the bars.

sets at both resolutions, indicating an absence of systematic difference between the two velocities.

For the low-resolution example (left), a constant velocity fit to the centroid position was performed over seven times, spanning an interval of about $9 \mathrm{hr}$. For the one time shown, the resulting velocity had a correlation coefficient of $\rho=0.73$ with $\overline{\boldsymbol{u}}_{a}$. For the other times in the series, the values for $\rho$ typically fall in the range $0.60 \lesssim \rho \lesssim 0.75$, indicating that the correlation between the two methods accounts for about half of the scatter when seven times are used.

The mean absolute deviation between the LCT and centroid speeds is $54 \mathrm{~m} \mathrm{~s}^{-1}$ when all partitions are given equal weight, but falls to $28 \mathrm{~m} \mathrm{~s}^{-1}$ when the mean is weighted by the flux in the partition. For the entire time series, the flux weighted absolute devia- tion is typically less than $35 \mathrm{~m} \mathrm{~s}^{-1}$. It is difficult to estimate the uncertainty in the LCT velocity, but by considering the scatter about the best-fit line we estimate that a typical uncertainty in the centroid velocity is $25 \mathrm{~m} \mathrm{~s}^{-1}$.

Calculating centroid velocities using linear fits over time intervals either substantially shorter or longer generally resulted in worse agreement between the LCT and centroid velocities. For longer intervals, the motion of the region probably cannot be well approximated as motion at constant velocity. For shorter times, variations in the partition result in larger errors (Barnes et al. 2005). This is evident from the larger uncertainties in smaller partitions, whose centroid location is more sensitive to changes in the partitioning.

For the high-resolution example on the right of Figure 7, a line was fit to the centroid position at 10 different times, spanning an
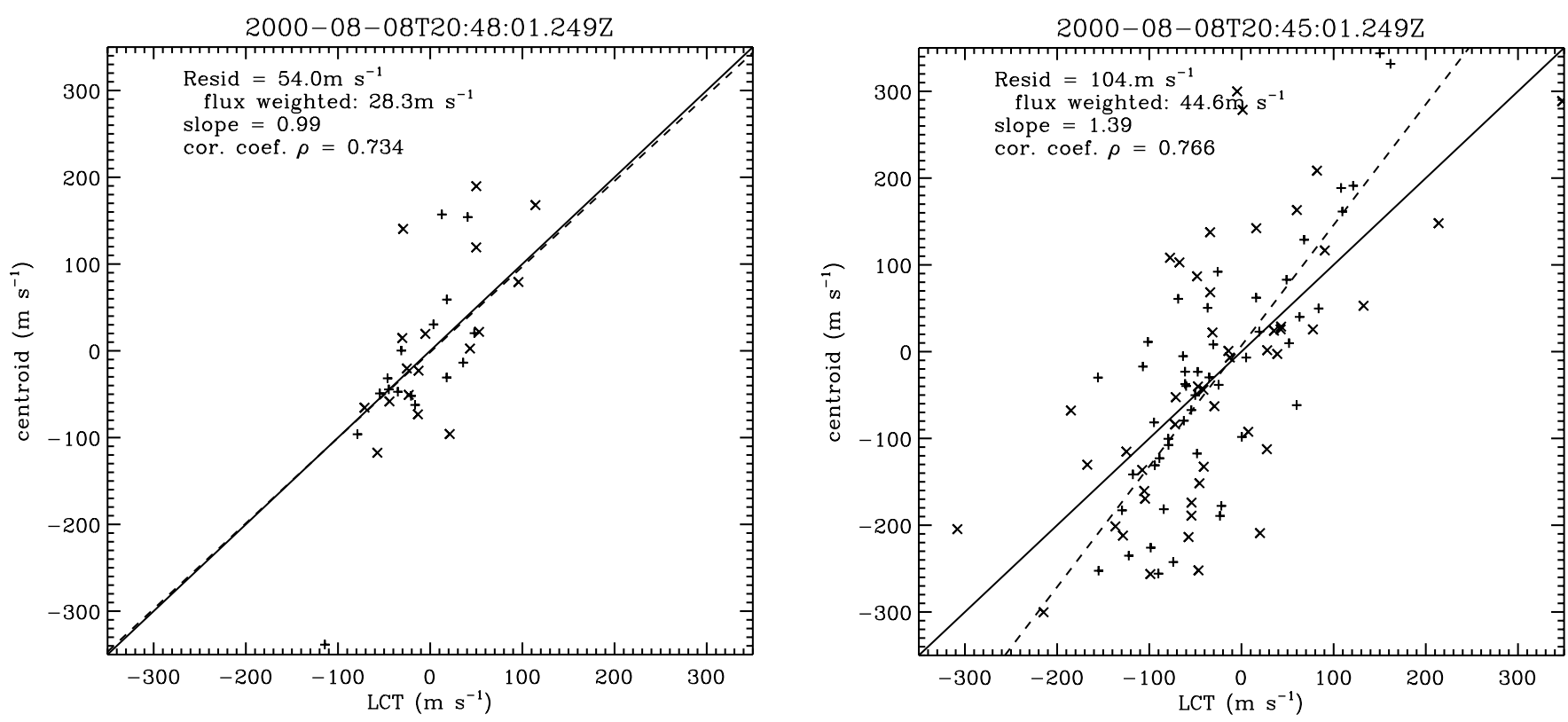

FIG. 7.-Two versions of the velocity of each partition. The centroid velocity, $d \overline{\boldsymbol{x}}_{a} / d t$, vs. the flux-weighted average of the LCT velocity at each pixel, $\overline{\boldsymbol{u}}_{a}$. The $x$-component of the each velocity is shown with a plus sign; the $y$-component is shown with a cross. The dashed line is the regression line. The high-resolution case (right) has a slightly higher correlation coefficient $(\rho=0.77)$ than the low-resolution case (left; $\rho=0.73)$, indicating better agreement between the two ways of determining the velocity, even though the mean absolute deviation is larger due to the generally larger speeds in the high-resolution case. 
interval of about $3.5 \mathrm{hr}$. The correlation coefficient for the case shown is $\rho=0.77$, and for other times the values of $\rho$ typically fall in the range $0.65 \lesssim \rho \lesssim 0.80$. There are more points than in the low-resolution case, owing to the larger number of partitions for high-resolution. As in the low-resolution case, fitting centroid sequence over longer or shorter time intervals resulted in worse agreement with LCT velocities.

The mean flux weighted absolute deviation for the case shown is $45 \mathrm{~m} \mathrm{~s}^{-1}$, and for other times it is typically $35-45 \mathrm{~m} \mathrm{~s}^{-1}$. Thus, even though the fit is better, the residual is larger. This is due to speeds that are typically larger than for the low-resolution data. The mean centroid speed for the low-resolution case is $119 \mathrm{~m} \mathrm{~s}^{-1}$, compared to a mean centroid speed of $271 \mathrm{~m} \mathrm{~s}^{-1}$ for the highresolution case. For the time series, it is typical for the mean speed calculated from the high-resolution data to be approximately double the mean speed for the low-resolution data.

There is a general tendency for LCT to yield higher speeds in each pixel of a high-resolution magnetogram than it does in a lowresolution magnetogram. In histograms of velocity magnitude (speed), the high-resolution data shows a tail extending from 0.5 to $1.2 \mathrm{~km} \mathrm{~s}^{-1}$ which is absent from low-resolution data. Berger et al. (1998) and Chae et al. (2004) demonstrated, using intensity images and MDI magnetograms, respectively, that the rms LCT velocity scales inversely with the width of the apodizing window. Since the minimum possible width of an apodizing window is fixed by the resolution, the high-resolution data will yield larger velocity.

\section{BENCHMARKING WITH ROTATING SUNSPOTS}

Section 3 tested the centroid velocities by comparing them to flux-weighted LCT velocities. In order to test the spin rate calculation, we turn to active regions containing rotating sunspots previously analyzed by BN03. Their study followed penumbral features in TRACE white light images in order to determine the rates of rotation over extended periods and as a function of radial distance from the umbral center. Annuli of a given radius, centered on the umbra, were unwrapped and formed into a stack plot of azimuthal angle versus time. Slanted streaks were then identified as moving features, and their slope used to determine a rotation rate. This measurement is sensitive to different structure and a different layer of the atmosphere than the magnetogram. We expect, however, that rotation of white-light features is indicative of a general rotation which should also be present in the magnetic field.

Figure 5 shows the partition from both low-resolution (top) and high-resolution (bottom) sequences of one case studied by BN03 (AR 9114). Although the regions and the naming do not coincide between the different data sets, P01 designates, in each one, the main leading flux concentration which includes the sunspot measured by BN03. Note that the region in the low-resolution sequence encompasses what are, in high-resolution, several other regions, such as P04, P08, and P09.

Comparisons can be made between the mean spin rate, $\bar{\omega}_{\mathrm{P} 01}$, in our region $\mathrm{P} 01$ and the sunspot rotation rates, $\dot{\theta}$, calculated in BN03. Figure 8 shows the velocity field, $\boldsymbol{u}-\overline{\boldsymbol{u}}$, within region P01. Each shows a counterclockwise (positive) sense of spin, especially near the northern boundary. It is evident from a comparison that the high-resolution data (bottom) produces larger velocities than the low-resolution data (top). Averaging the azimuthal rotation rate, $u_{\phi} / r$ within each centroid-centered annuli in the image gives the rotation profiles shown in Figure 9. For comparison we plot the rotation rate profile, $\dot{\theta}(r)$, from Figure $5 \mathrm{~b}$ of BN03. Their stack plots made from TRACE white light images were less sensitive to umbral motions; consequently, their profile decreases at small
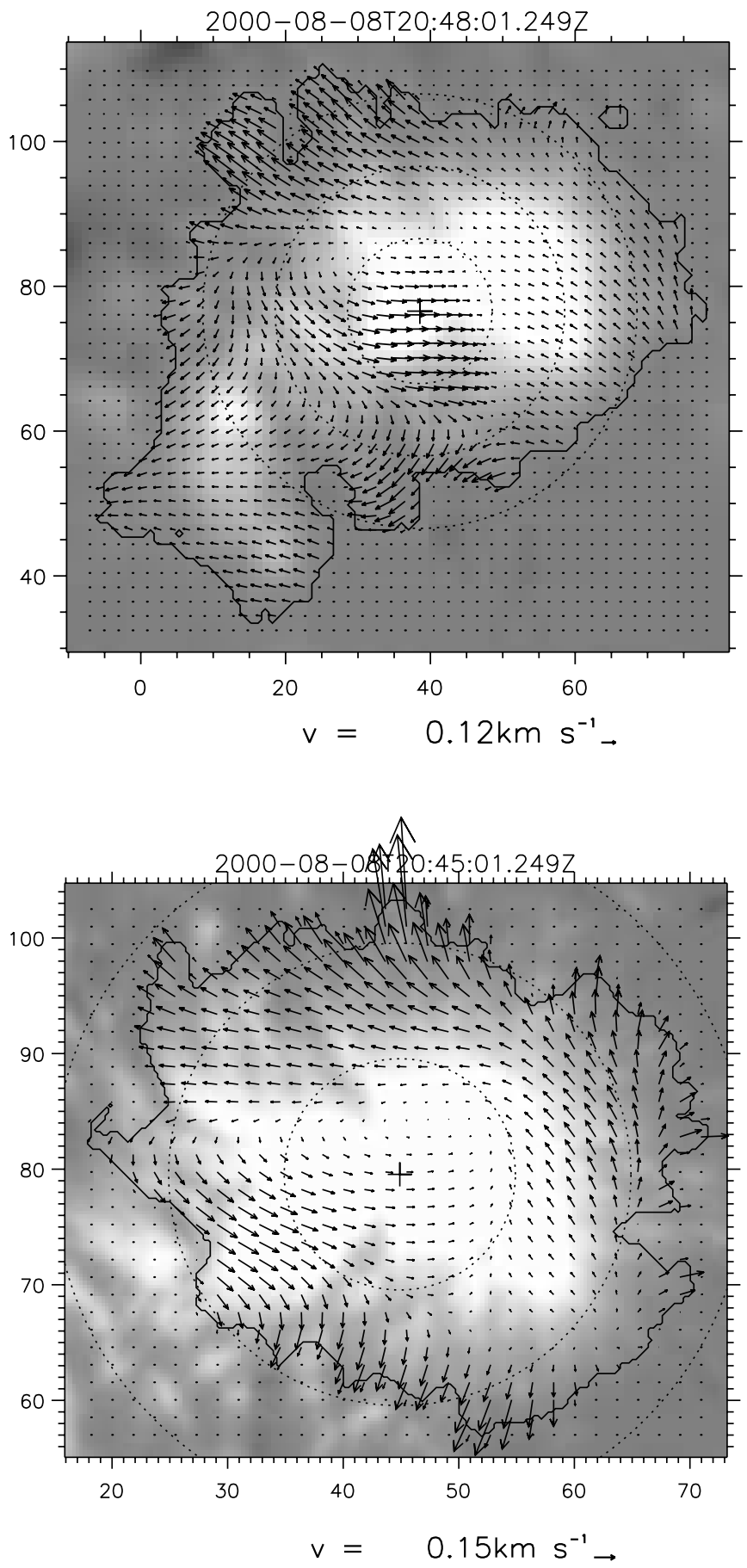

FIG. 8.-Velocity vectors overlaid on the low-resolution magnetogram (top) and high-resolution magnetogram (bottom). The reference arrows at the bottom of each panel are in terms of $\mathrm{km} \mathrm{s}^{-1}$. The plus signs mark the flux-weighted centroids, and dotted circles are drawn at radii of $10^{\prime \prime}, 20^{\prime \prime}$, and $30^{\prime \prime}$ from centroid.

radii. The LCT-derived profile, especially at high resolution, suggests rigid rotation at central radii.

It is evident in Figure 8 that the location of the centroid is not precisely the same in the high- and low-resolution cases. This is primarily a result of the inclusion of additional flux in the lowresolution partition for source $\mathrm{P} 01$ that is part of separate partitions in the high-resolution case. It is to be expected that the center of the sunspot as identified in BN03 will be at yet a third location. However, by considering different ways of partitioning the lowresolution data, we find that the mean spin rate, $\bar{\omega}_{\mathrm{P} 01}$, and hence the spin helicity are not greatly impacted by shifts in the centroid 

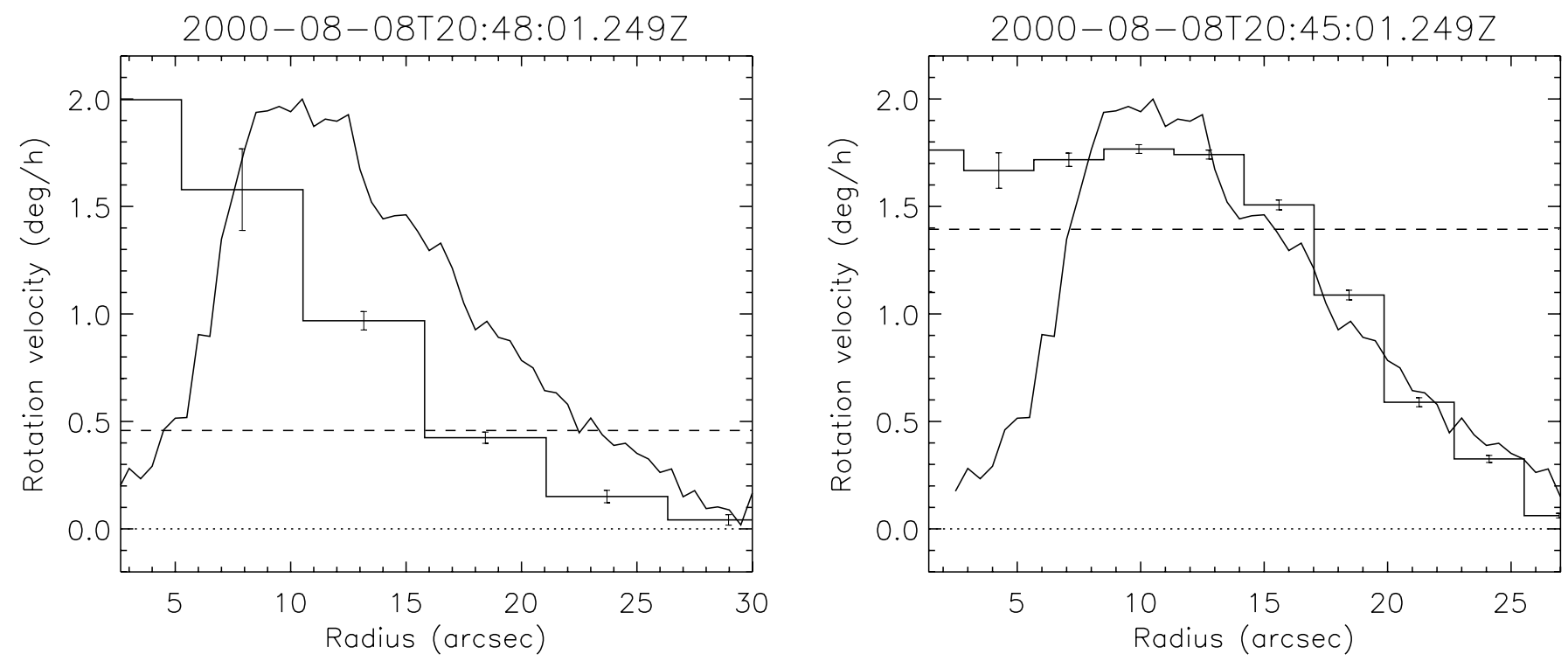

FIG. 9.-Profiles of rotation rate vs. radius for region P01 of region AR 9114 using low-resolution magnetogram partition and velocity (left) and high-resolution magnetogram and velocity (right). The vertical bar in the plot is an error in estimating the rotation rate. The rotation rate is measured at fixed radius (as shown in Fig. 8) with flux weighted centroid as a center. The horizontal dashed line is the mean rotation rate, $\bar{\omega}_{\mathrm{P} 01}$, defined according to expression (14).

location. This is particularly encouraging because it implies that our results are not sensitive to small changes in the partitioning, as are inevitable when tracking a flux concentration over a long time interval.

Figure 9 shows the curve from $\mathrm{BN} 03$ compares more favorably to the high-resolution data set than the low-resolution set. The former appears to match white light data outside $10^{\prime \prime}$, while the latter appears to be systematically lower by a factor of 2 . The helicity injection will ultimately depend on the single rotation rate, $\bar{\omega}$, derived from the velocity field according to expression (14). The value for each case is plotted as a horizontal dashed line. The high-resolution value, $\bar{\omega} \simeq 1.4^{\circ} \mathrm{hr}^{-1}$, is a fair representation of the overall profile. The low-resolution value, $\bar{\omega} \simeq 0.5^{\circ} \mathrm{hr}^{-1}$, is lower partly due to slower rotation of the outlying portions, absent from the high-resolution partition, and partly due to its generally lower velocities. BN03 summarize their profile with an "average" rate of $\dot{\theta}_{\mathrm{av}} \sim 1.1^{\circ} \mathrm{hr}^{-1}$ and a "penumbral" rate for $\dot{\theta}_{\mathrm{pen}} \sim 2^{\circ} \mathrm{hr}^{-1}$. The former is intended to represent the overall profile, in a similar spirit to our quantity $\bar{\omega}$. We therefore use this value in our comparison.

The rotation rate, $\bar{\omega}$, is directly related to helicity injection. Figure 10 compares this to the rates reported in $\mathrm{BN} 03$ for the leading sunspot in AR 9114. During the long, low-resolution data set (left) $\bar{\omega}$ evolves a similarly to $\dot{\theta}_{\text {av }}$ from BN03: gradually increasing until $t \sim 55$, after which the rotation slows to zero by $t \sim 80$. The tendency of the low-resolution data to underestimate the rotation rate, noted for the profile Figure 9, appears to persist for the entire sequence.

The high-resolution data (right), on the other hand, provide a superior match to the measurements of BN03. Over its short duration both the TRACE-measured rotation rate and the LCT rate remain steady at a bit over $1^{\circ} \mathrm{hr}^{-1}$. Indeed, $\bar{\omega}$, remains systematically higher than $\dot{\theta}_{\mathrm{av}}$, as it did in the profile. The plus signs in the lower panels in Figure 10 show the spin helicity flux attributable to the rotation of $\mathrm{P} 01$, namely $-\bar{\omega}_{\mathrm{P} 01} \Phi_{\mathrm{P} 01}^{2} / 2 \pi$. The helicity
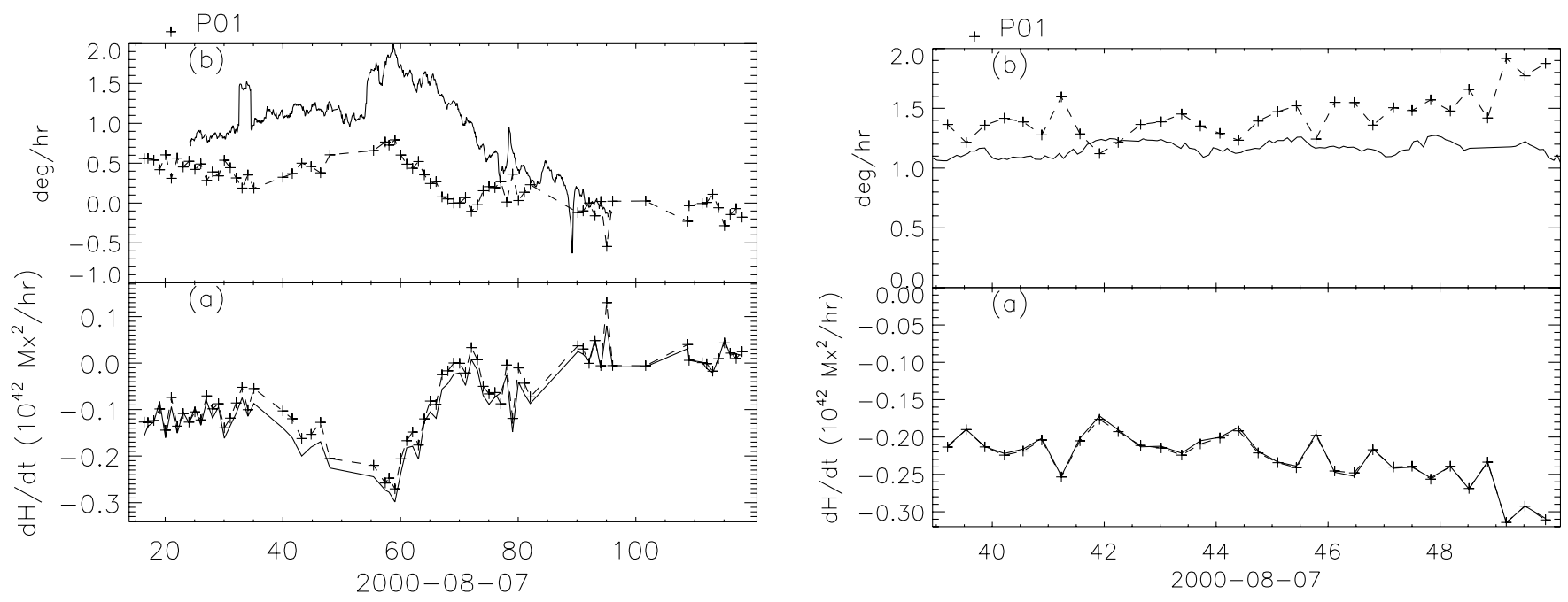

FIG. 10.- (a) Spin helicity and $(b)$ rotation rate for the positive region P01, using the sequence of low-resolution (full-disk) magnetograms (left) and high-resolution magnetograms (right). These are plotted vs. time in hours after 00:00 UT August 07. The results of the LCT tracking are indicated by plus signs. The solid curves in the $b$ panels are the "average" rotation rate of the white-light sunspot, taken from BN03, while the solid curves in the $a$ panels are the total spin helicity flux, $\dot{H}_{\text {sp }}$, for all flux regions in the magnetogram. 


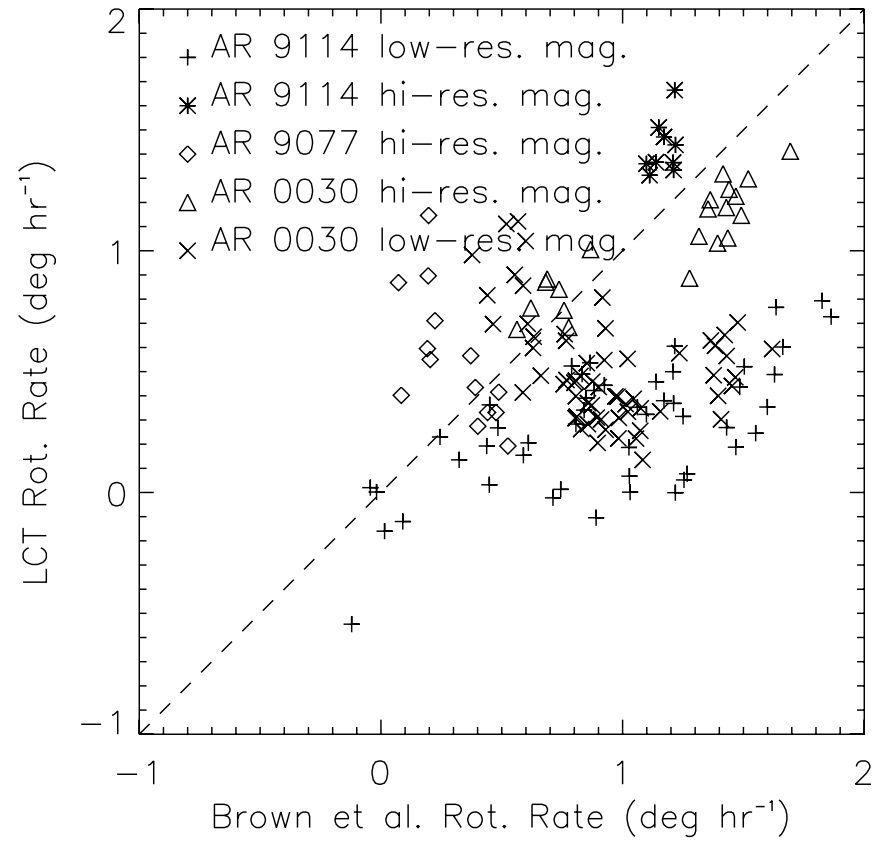

FIG. 11.-Comparisons of rotation rates derived by BN03 and the present LCT analysis. High-resolution data sets for three different ARs and low-resolution data sets for two of them are plotted as different symbols ( plus signs: low-resolution 9114; asterisks: high-resolution 9114; diamonds: 9077; triangles: high-resolution 10030; and crosses: low-resolution 10030).

decomposition finds rotation rates for each of the regions, not just the large sunspot. Each of these contributes to the total spin helicity flux, plotted as the solid curve. The very close agreement between this total and the contribution of P01 alone is due to the overwhelming dominance of that single region in the total flux (see Fig. 6). The underestimation of rotation rate in the lowresolution sequence (left) originates in an underestimation in the helicity flux, by at least a factor of 2 in the present case. Since spin helicity is a contributor to the total helicity flux we conclude that using low-resolution magnetograms will result in a systematic error in its calculation.

We believe that the underestimation in low-resolution data is not simply a result of the longer $(1 \mathrm{hr})$ time difference and broader $\left(7^{\prime \prime}\right)$ apodizing window size used. To establish this we computed $\bar{\omega}_{\mathrm{P} 01}$ from low- and high-resolution data using a range of time steps, down to 15 minutes, and apodizing window sizes, down to $5^{\prime \prime}$ for low and 3.6" for high resolution. For a given data set, the spin rate scaled inversely with apodizing window, in agreement with the studies of Berger et al. (1998) and Chae et al. (2004). When, however, both data sets are analyzed using the same apodizing window, 6 " for example, the high-resolution data yields a rotation rate almost twice as high as low-resolution data. The most likely reason for this discrepancy is the tendency, previously noted, for LCT to produce higher velocity values from high-resolution MDI data. Therefore, in order to reduce noise in the velocity measurement we continue to use $1 \mathrm{hr}$ and $7^{\prime \prime}$ for LCT on low-resolution data.

We also managed to rule out the possibility that the saddle point-merging step in the partitioning led to the discrepancy in the rotation rate. We did this by experimenting with saddle pointmerging values other than $300 \mathrm{G}$. Using 100 or $200 \mathrm{G}$ instead yielded partitions of the low-resolution data resembling those of high-resolution data, but still with systematically lower spin rates.

Of the other cases considered by BN03 we obtained highresolution MDI sequences for ARs 9077 and 10030, and analyzed them in the same manner just described. In both cases the rotation

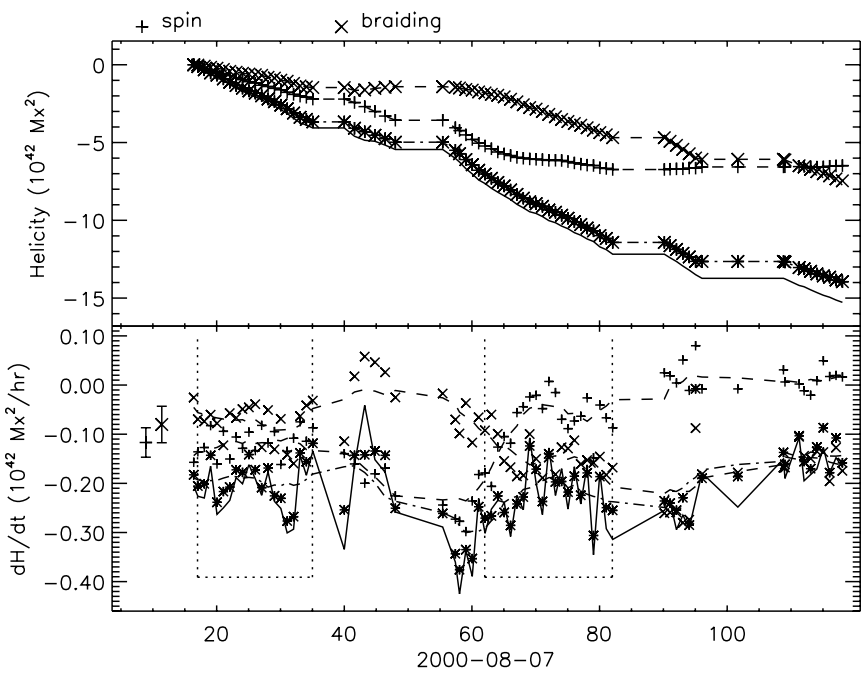

FIG. 12.- Summary of the helicity flux (bottom) and its integral (top) into AR 9114 using the low-resolution data set plotted vs. time in hours from 00:00 UT on August 07 . The total is indicated by a solid line, its decomposition into spin and braiding components is indicated by plus signs and crosses, respectively, and the sum of these is shown as asterisks. Vertical dotted lines in the bottom panel designate the interval over which $\dot{H}$ was averaged. The values averaged over the first interval are indicated by crosses and plus signs to the left of the actual data. Each of the average values is bracketed with an error bar equal to the error in a single measurement.

rates of the sunspot-enclosing region agreed with BN03 at a level similar to the high-resolution case of 9114 just described. Figure 11 summarizes the comparisons by plotting $\bar{\omega}$ versus $\dot{\theta}_{\text {av }}$ from BN03, for each time in our sequence. Since our values are spaced at intervals of $1 \mathrm{hr}$ in the low-resolution and 20 minutes in the high-resolution magnetograms, we averaged the BN03 data and high-resolution data points over $1 \mathrm{hr}$ interval for the purposes of comparison. The pairs of corresponding rotation rates lie in the general vicinity of equality (dashed line) for the high-resolution cases (triangles, diamonds, and asterisks). The low-resolution values (plus signs and crosses) are almost all underneath the dashed line, indicating that the rotation rate is systematically underestimated. The data cluster about a line, but not tightly enough to be characterized as underestimation by a simple factor.

Based on these data we conclude that the nominal rotation rate, $\bar{\omega}$, corresponding to spin helicity injection, is a reasonable proxy for the rotation rate of a sunspot. Conversely, we also conclude that observed sunspot rotation at $\dot{\theta}$ is accompanied by a helicity flux $\dot{H}=-\dot{\theta} \Phi^{2} / 2 \pi$. This will not, however, be the only contribution to the helicity.

\section{DECOMPOSITION OF HELICITY FLUX}

\subsection{AR 9114}

Figure 12 shows the total spin helicity (plus signs) and braiding helicity (crosses) from the decomposition of the longer, lowresolution sequence for AR 9114 discussed in $\S 4$. (The total spin helicity flux, $\dot{H}_{\text {sp }}$ matches the solid line in the left of Fig. 10a.) The sum of these contribution is shown with an asterisk, while the exact total, from expression (9), is shown by a solid curve. The last two are not equivalent due to the higher order terms neglected when approximating braiding helicity, equation (15), with equation (16). They are, however, very close suggesting that the neglected terms are not very significant in the present partition.

Our calculations yield helicity fluxes between pairs of magnetograms closely spaced in time $(0.25-1 \mathrm{hr}$ apart). There are, unfortunately, a few gaps exceeding $4 \mathrm{hr}$ in the data sequence. We 
TABLE 1

Summary of the ARs for which Helicity Decomposition Was Performed

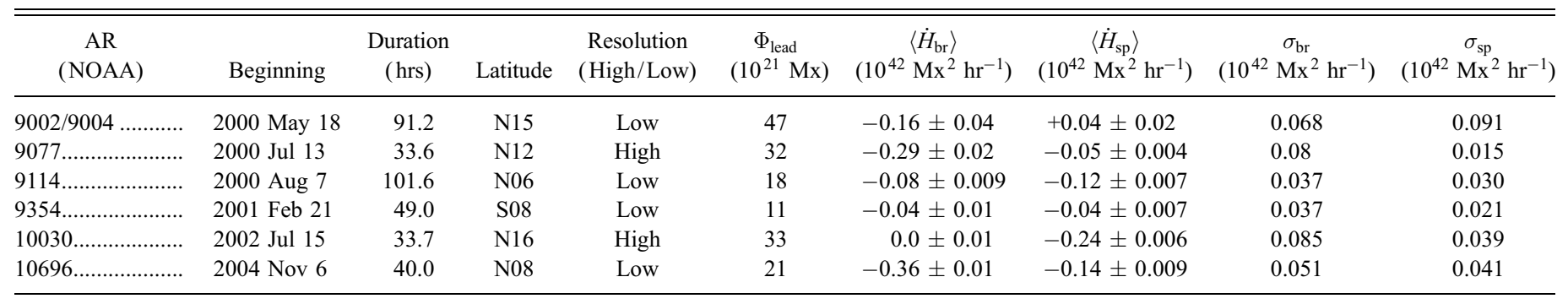

Notes.- Helicity flux rates were averaged during a stable period indicated in Figs. 12 and 13. If more than one period was considered, the table lists results from the first.

choose not to apply LCT across the gaps. The partitioning does appear relatively consistent across them; however, we do not attempt to calculate either braiding or spin helicity during the gaps. The net helicities, shown in the top panel, are found by integrating helicity fluxes. We do not perform this integral across the gaps, but instead take the conservative approach of beginning the postgap integral at the value from before the gap. For cases of relatively steady injection this will underestimate the net helicity injection.

Plots of the spin and braiding flux contribution, in the lower panel of Figure 12, are accompanied by a smoothed curve (dashed line) calculated using a nine-point boxcar average. Under the assumption that the true helicity flux within an AR varies somewhat slowly, we consider the deviation from this curve by the individual measurements ( plus signs and crosses) to represent intrinsic noise in the technique. We believe the principal source of this noise is the partitioning algorithm (Barnes et al. 2005), and quantify by $\sigma_{\mathrm{sp}}$ and $\sigma_{\mathrm{br}}$ the rms deviation from the smoothed curve. (The window width of nine points was chosen by eye; other reasonable choices yield almost the same noise levels). In the present case we find $\sigma_{\mathrm{sp}}=0.037$ and $\sigma_{\mathrm{br}}=0.030$, in units of $10^{42} \mathrm{Mx}^{2} \mathrm{hr}^{-1}$. These values are indicated by error bars to the left of the data in the lower panel.

To further clarify the roles of spin and braiding helicity flux in the development of AR 9114, we have averaged them over two distinct intervals. During the first interval, $t \in(17,35)$, we find $\dot{H}_{\text {sp }}=-0.12 \pm 0.007$ and $\dot{H}_{\mathrm{br}}=-0.08 \pm 0.009$, once again in units of $10^{42} \mathrm{Mx}^{2} \mathrm{hr}^{-1}$. These values are indicated within the error bars; however, since they are means, their error is much smaller than the error bars, which depict the error in individual measurements. We may conclude that the spinning of the sunspot in P01 is the primary contributor of helicity, but the motion of surrounding flux contributes helicity of the same sign. At this point we should recall that the spin helicity is underestimated, by at least a factor of 2, by these low-resolution magnetograms. If the braiding helicity is more accurately calculated then the spin helicity contribution is therefore even more dominant than our numbers suggest.

The second interval, $t \in(62,82)$, is a time when the rotation rate of the sunspot is slowing down. The declining spin seems to be compensated by an increasing braiding contribution. The averages during this interval are $\dot{H}_{\mathrm{sp}}=-0.08$ and $\dot{H}_{\mathrm{br}}=-0.14$. If we assume the spin helicity is once again underestimated, then the braiding is at least comparable to spin in the helicity injection. Notably both contributions are always for the same sign for this AR.

By the end of the $100 \mathrm{hr}$ sequence the net helicity of AR 9114 has changed by at least $\Delta H=-15 \times 10^{42} \mathrm{Mx}^{2}$; the change could be greater since we did not include possible contributions during the data gaps. It appears that the helicity flux was almost equal parts spinning and braiding in this particular case. During this time the sunspot penumbra rotated $\simeq 75^{\circ}$, spinning as fast as $3^{\circ} \mathrm{hr}^{-1}$ at times (BN03). The LCT-derived rotation rate, $\bar{\omega}$, is typically smaller than this, and its integral is $\simeq 21^{\circ}$ over the entire time. Putting this counterclockwise turn into the $1.1 \times 10^{22} \mathrm{Mx}$ of flux composing region $\mathrm{P} 01$ will introduce $\Delta H \simeq-7 \times 10^{42} \mathrm{Mx}^{2}$ all by itself.

\subsection{Other Cases}

We have analyzed five other data sets of evolving ARs to find their spin and braiding helicities. These analyses, six in all, are summarized in Table 1. Time series of most of them are plotted in Figure 13, following the style of Figure 12. Five of the six ARs (9004, 9114, 9077, 9354, and 10030) include rotating sunspots analyzed by $\mathrm{BN} 03$.

Even this small, and nonrepresentative, sample includes an extraordinary diversity of behaviors. In some regions (9114 and 10030) spin dominates braiding, while in others braiding dominates spin (9002/9004, 9077, and 10696). The spin and braiding contributions are sometimes of the same sign $(9114,9354$, and $10696)$, while they can also oppose each other (9002/9004). In the case of the complex 9002/9004 there is an initial period of braiding dominance $[t \in(5,20)$ listed in the table] followed by a period of spin dominance $\left[t \in(40,60)\right.$, where $\dot{H}_{\mathrm{sp}}=+0.35$ and $\left.\dot{H}_{\mathrm{br}}=-0.10\right]$, and then a final period of braiding dominance. Since the contributions in this case are also of opposite sign the total helicity flux changes sign each time the roles reverse. The total helicity injected in the end is small, and of the sign opposite to the helicity injected by the rotating sunspot.

The partitioning errors are generally small enough so that averaging $\sim 10$ measurements will yield a mean helicity flux with error $\lesssim 2 \times 10^{40} \mathrm{Mx}^{2} \mathrm{hr}^{-1}$. This attests to the stability of the partitioning, since its fluctuations are believed to be the greatest source of noise (Barnes et al. 2005). For this reason, and also due to their generally higher velocities, the high-resolution sequences do not always have the lowest noise (see e.g., AR 10030).

\subsection{AR Complex 9002/9004}

The case of AR 9002/9004 is a particularly interesting one, since it comprises a pair of ARs from which one (AR 9004) is emerging. The partition of the complex, shown in Figure 14, has one large region, $\mathrm{P} 01$, which includes the leading sunspot of $\mathrm{AR}$ 9002. The smaller AR to the south, AR 9004, includes the negative spot (N03) which BN03 observed to be rotating clockwise. The sunspot rotation is increasing steadily throughout May 18, up to $2^{\circ} \mathrm{hr}^{-1}$, after which it decreases more rapidly. We find that both polarities of AR 9004 (N03 and P02) spin. The positive polarity, P02 begins a rapid clockwise spin at about 16:00 May 19 $(t=40$; see Fig. 15$)$, which changes steadily into counterclockwise spinning at $\bar{\omega} \simeq 1^{\circ} \mathrm{hr}^{-1}$. The polarities of the older region (AR 9002), in contrast, have no measurable rotation at all. 

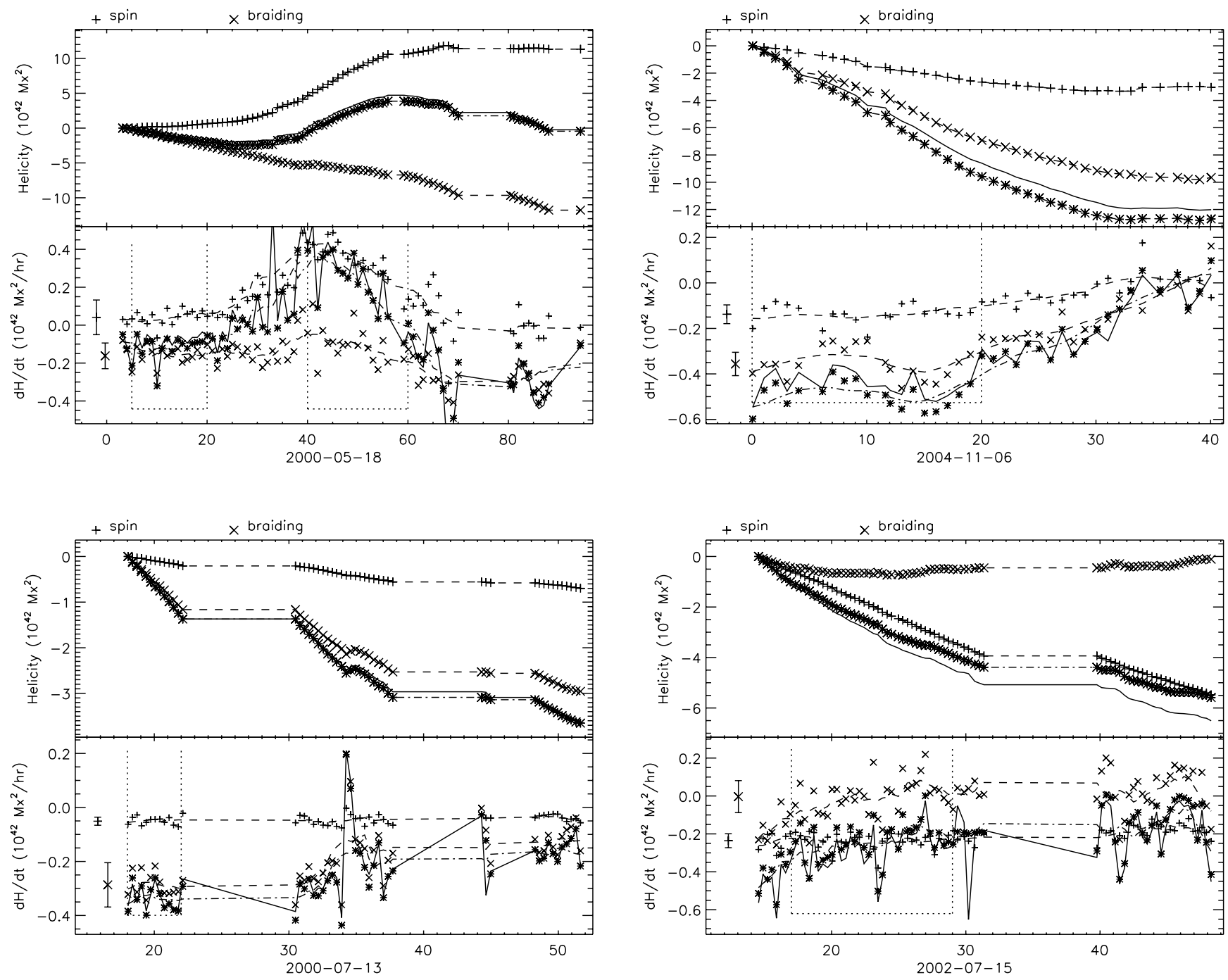

FIG. 13.-Time histories of helicity injection for $9002 / 9004$ (top left), 10696 (top right), 9077 (bottom left), and 10030 (bottom right). Each plot has the same format as Fig. 12.
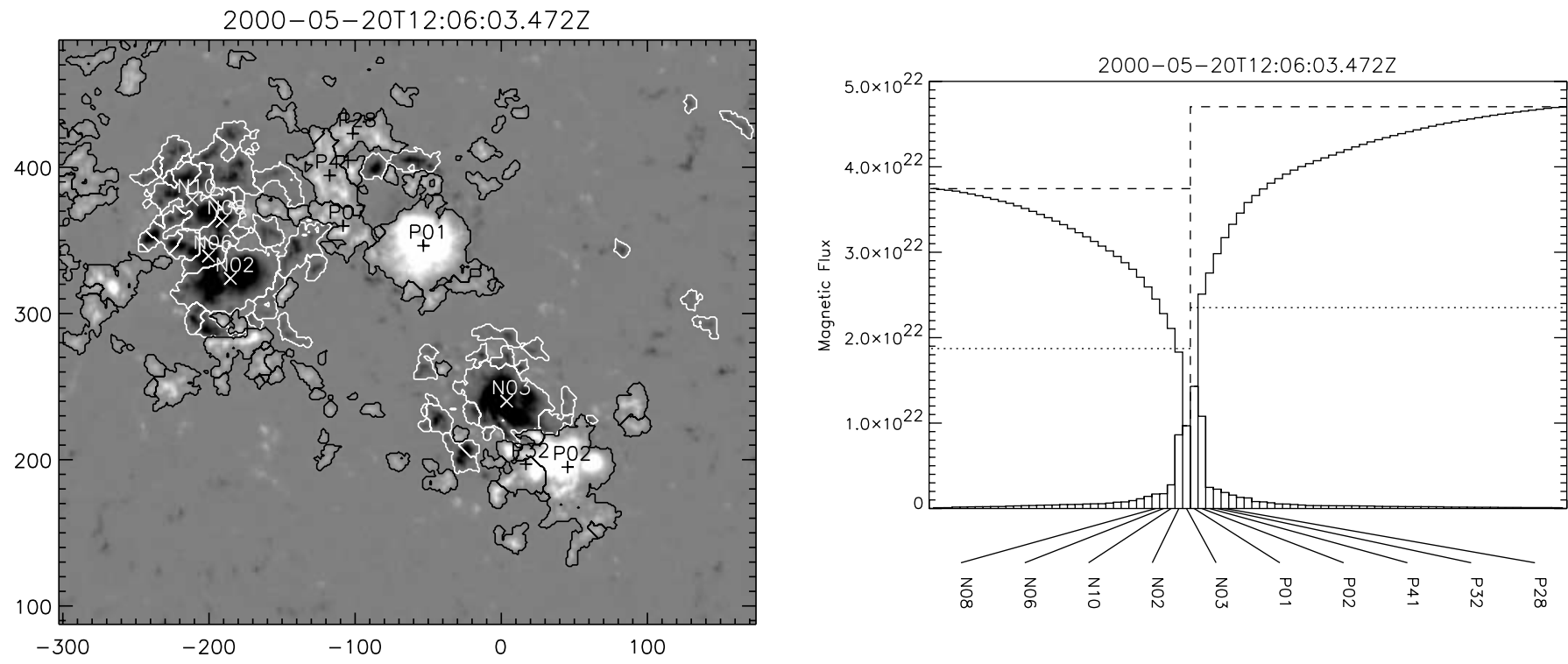

FIG. 14.-Partitioning of the magnetogram containing ARs 9002/9004. On the left is the MDI magnetogram (scaled between $\pm 1000 \mathrm{G}$ ), with the partition boundaries represented. The centroids of the largest regions are designated and labeled. On the right are histograms of the partition fluxes, as in Fig. 6. 


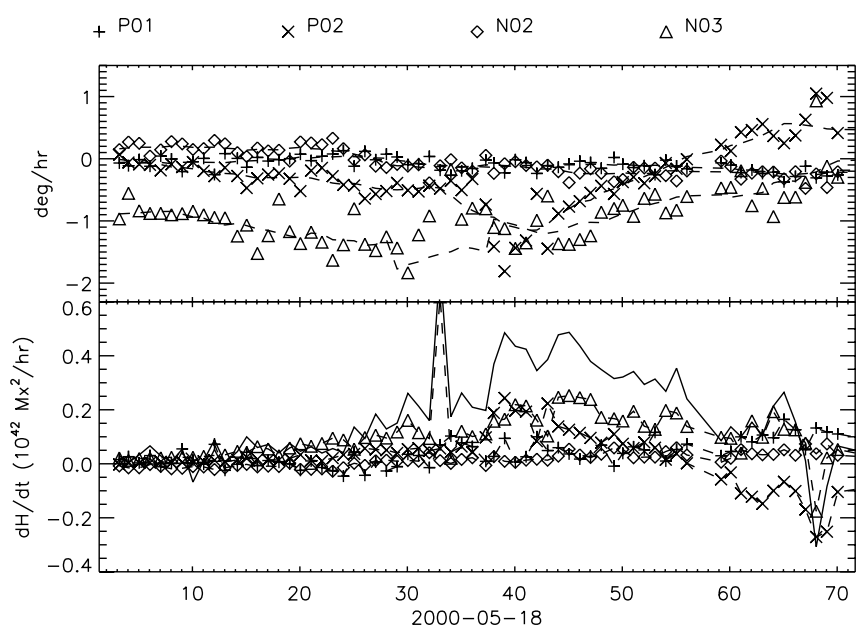

FIG. 15.-Spin rates, $\bar{\omega}_{a}$ (top), and helicity fluxes, $\dot{H}_{\mathrm{sp}}^{a}$ (bottom), from several of the larger regions in 9002 and 9004 , labeled in Fig. 14. These are regions from 9002 are P01 (plus signs) and N02 (diamonds) and from 9004 are P02 (crosses) and N03 (triangles). Each region's rotation rate is accompanied by a dashed curve smoothed using a nine-point smoothing window. The solid curve in the lower panel is the total spin helicity, $\dot{H}_{\text {sp }}$, from all sources.

The flux emergence in AR 9004 is slightly more complex than the basic bipolar scenario. The region was at least partly formed when it rotated onto the disk on May 15. As it became clearly visible on May 16 and 17, it appeared stable and closely aligned with the equator $\left(\theta=180^{\circ}\right)$. Then on May 18 the fluxes in its polarities began increasing, as shown in Figure 16. The positive and negative fluxes both increase approximately linearly, but beginning and ending at slightly different times: the positive polarity from $t=20$ to 60 (hours from 00:00 May 18) and the negative from $t=10$ to 55. After reaching their maxima $\left(\max \Phi_{+}=15 \times\right.$ $10^{21} \mathrm{Mx}$ and $\max \Phi_{-}=12 \times 10^{21} \mathrm{Mx}$ ), they begin to break up, causing the flux in the large regions to decrease. During their linear phase the fluxes change at about the same rates: $\dot{\Phi}_{+}=0.14 \times$ $10^{21} \mathrm{Mx} \mathrm{hr}^{-1}$ and $\dot{\Phi}_{-}=0.13 \times 10^{21} \mathrm{Mx} \mathrm{hr}^{-1}$.

The separation between the centroids of the polarities decreases at $\dot{d}=-150 \mathrm{~m} \mathrm{~s}^{-1}$ until about $t=20(d=33 \mathrm{Mm})$, and thereafter increases at the leisurely rate of $\dot{d}=37 \mathrm{~m} \mathrm{~s}^{-1}$. The converging phase occurs at a tilt angle of roughly $\theta=150^{\circ}$, a sense consistent with Joy's law angle. As the flux emergence begins, at $t=20$, the polarities begin to circle about one another until they are oriented almost perpendicular to the equator $\left(\theta=90^{\circ}\right)$. It is this clockwise braiding motion that is responsible for much of the negative braiding helicity injection.

The approaching polarities, rapid rotation away from the Joy's law tilt, and opposing spin and braiding injections all suggest that the evolution is driven by a kink instability (Linton et al. 1998). Relative motions of polarities have been used previously to infer the kinked flux tube axis (Pevtsov \& Longcope 1998; López Fuentes et al. 2000). Our helicity decomposition provides additional information about the process. The different time histories of the spin and braiding rates is difficult to reconcile with a simple rigid motion of a kinked tube across the photosphere. The delayed spinning of the positive pole suggests that the kinking had occurred on the following side of the AR and then propagated along the flux tube. This propagation would naturally produce an opposing spin in the other polarity (Longcope \& Welsch 2000), which does eventually occur. It is possible that a portion of the tube which is twisted and writhed is emerging, but that the twist propagates into the atmosphere through the torque-imbalance mechanism of Longcope \& Welsch (2000). If this is so, then the virtual can-

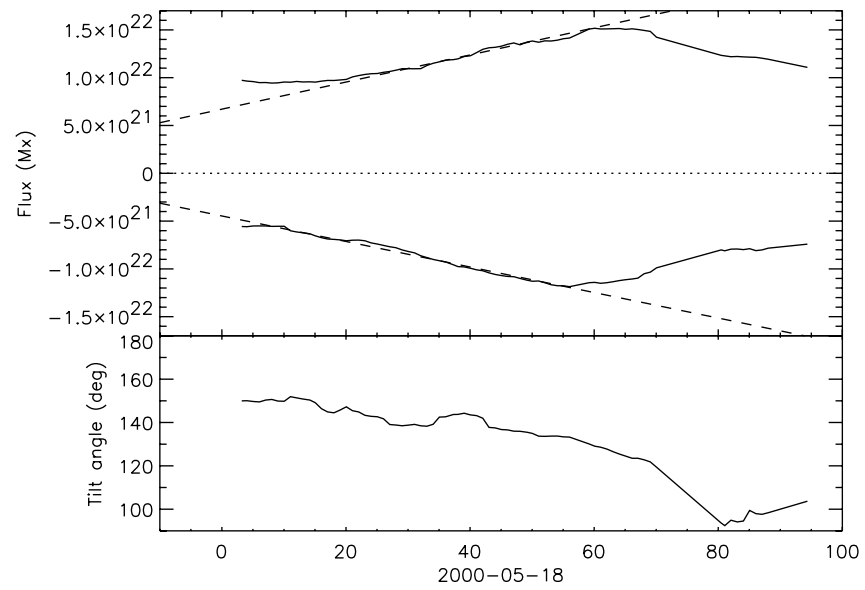

FIG. 16. - Evolution of flux (top) and polar separation (bottom) in AR 9004. These are calculated within the tangent plane and include all of the significant regions of each polarity composing that AR. Dashed lines are the linear fits used to calculate the rates of change $\dot{\Phi}_{ \pm}$reported in the text.

cellation of spin and braiding helicity is consistent with a kink caused by the flux tube kink instability.

\section{DISCUSSION}

The foregoing has described a practical method of decomposing helicity flux into spin and braiding contributions due to motions internal and external, respectively, to specified photospheric regions. If a source region is the end of an isolated subphotospheric flux tube and its motion is horizontal, then spin comes principally at the expense of its twist, and braiding helicity can be intuitively attributed to changes in its writhe. The decomposition provides a definition of an averaged rate of spin within a complex region undergoing complex internal motion.

This decomposition can be compared to a characterization of the helicity flux distribution recently proposed by Pariat et al. (2005). That method produces a pseudodensity, $G_{\theta}(x, y)$, on the photosphere whose integral yields the total helicity flux. It is not a genuine density of helicity flux, whose exact construction would require nonlocal knowledge in the form of details of the mapping through the coronal field (Pariat et al. 2005). Instead it is a convenient proxy, which suffers from fewer artifacts than the alternative, $G_{A}=-2\left(\boldsymbol{u} \cdot \boldsymbol{A}_{P}\right) B_{z}$. (It is easily seen from eq. [9] that the integral of this quantity also yields the total helicity flux.) To the extent that $G_{\theta}(x, y)$ possesses the properties of a genuine density, its integral over a photospheric region $\mathcal{R}_{a}$, would seem to yield an analog of the spin helicity of that region. It can be readily seen, however, that the sum of such spin helicities will equal the total helicity flux; there is no analog of braiding helicity in such a scheme. This points to the difficulties inherent in defining a local density for the nonlocal quantity of relative helicity, and to the differences between the pseudodensity of Pariat et al. (2005) and our spin/braiding decomposition.

We have developed a novel partitioning algorithm which permits us to apply our method to long time sequences of AR observations. The algorithm defines regions using a gradient-based tessellation matched to an evolving reference partition. Local correlation tracking (LCT) is used to derive a velocity with which the reference partition is evolved. Using time sequences of lowresolution and high-resolution MDI data, we verify that the partitions follow the photospheric flow. The agreement also serves to test the braiding helicity component of the decomposition.

Magnetogram partitioning is not a unique process and the output of the algorithm depends on choice of its parameters. We show 
theoretically that braiding helicity in one partitioning will be designated as spin helicity in a coarser partitioning. This ambiguity is natural in light of the imprecise segregation of subphotospheric field into flux tubes. In reality it is probably not possible to assign each bit of photospheric flux to a distinct flux tube, so it should not be possible to clearly segregate helicity according to twist and writhe of hypothetical tubes. In spite of this, the flux tube concept has proven useful in explaining the existence and behavior of active regions, at least on gross scales. We expect our decomposition to be valid at roughly the same level since a few large partitions typically account for the majority of all magnetic flux and helicity flux.

To test our measurement of spin rate we perform decompositions on ARs containing rotating sunspots previously studied using TRACE white-light observations by Brown et al. (2003, referred to as BN03). This represents a novel cross-calibration of LCT-derived helicity fluxes. We find that spin helicity rates computed from high-resolution MDI data agree well with sunspot rotation rates, both as functions of time and of radius. The spin rate derived from the partition is found directly from a helicity flux. Its agreement with penumbral rotation rates confirms that a rotating sunspot injects magnetic helicity into the corona at a rate $\sim \dot{\theta} \Phi^{2} / 2 \pi$.

Spin rates derived from low-resolution MDI magnetograms agree with sunspot rotation curves but appear to systematically underestimate their magnitude. This underestimation is probably due to LCT velocities being systematically lower when lowresolution magnetograms are used. We also found, in $\S 3$, that the lower LCT velocities produced lower centroid velocities. This leads us to expect both spin and braiding helicity fluxes to be underestimated by low-resolution magnetogram sequences. Since many of the helicity calculations reported in the literature have used low-resolution MDI data (necessary for long time sequences) these have probably been subject to similar underestimation.

We applied our algorithm to sequences of magnetograms for six different active regions. In order to include several cases extending over many days, it was necessary to use low-resolution data and accept its tendency to underestimate helicity fluxes.
Among our cases we observed some where spin was the dominant source and others where braiding was. In some cases the two contributions were of the same sign while in others they opposed one another. One case in particular had opposing signs of spin and braiding whose differing time history appeared consistent with a current-driven kink instability within the submerged flux tube. In spite of the significant spinning and braiding there was little net helicity injection in this case. This would be expected if the kink instability were converting twist helicity to writhe helicity without altering their sum.

The six cases we treated were intended to illustrate the decomposition technique, rather than provide a statistical picture of helicity flux. Nevertheless, it showed that coronal helicity probably does not originate from either the twist or writhe of subphotospheric flux tubes alone. Instead it appears that either or both kinds of helicity can contribute in different cases. It is still possible that a general pattern exists, perhaps involving the age or life history of the AR. A more extensive study could eventually reveal such a pattern.

The decomposition presented here may also prove useful in modeling the storage of coronal energy in AR fields. Indeed, the analysis of AR 10696, presented in $\S 5$, has already been used in this way. This region was the site of a large flare (GOES class X2) and CME late on November 7. The helicity decomposition reveals that motions prior to that event injected helicity primarily through braiding. This fact was used by Longcope et al. (2007) to model the energy storage. They were able to produce a model in which the relative motions of the AR's flux concentrations (essentially a shearing) stored the energy prior to flaring. Had the helicity decomposition shown significant spinning it would have been necessary to model the internal motions of the concentrations as well.

We thank Daniel Brown for providing us the data from BN03. This work was funded in part by the DoD under the MURI program and by the Air Force Office of Scientific Research under contract FA9550-06-C-0019.
Barnes, G., Longcope, D. W., \& Leka, K. D. 2005, ApJ, 629, 561

Berger, M. A. 1984, Geophys. Astrophys. Fluid Dyn., 30, 79

1988, A\&A, 201, 355

. 1991, A\&A, 252, 369
Berger, M. A., \& Field, G. B. 1984, J. Fluid Mech., 147, 133

Berger, M. A., \& Prior, C. 2006, J. Phys. A, 39, 8321

Berger, T. E., \& Lites, B. W. 2003, Sol. Phys., 213, 213

Berger, T. E., Loefdahl, M. G., Shine, R. S., \& Title, A. M. 1998, ApJ, 495, 973

Brown, D. S., Nightingale, R. W., Alexander, D., Schrijver, C. J., Metcalf, T. R.,

Shine, R. A., Title, A. M., \& Wolfson, C. J. 2003, Sol. Phys., 216, 79 (BN03)

Chae, J. 2001, ApJ, 560, L95

Chae, J., Moon, Y.-J., \& Park, Y.-D. 2004, Sol. Phys., 223, 39

Chae, J., Wang, H., Qiu, J., Goode, P. R., Strous, L., \& Yun, H. S. 2001, ApJ, 560,476

Choudhuri, A. R., \& Gilman, P. A. 1987, ApJ, 316, 788

Démoulin, P., \& Berger, M. A. 2003, Sol. Phys., 215, 203

Démoulin, P., Mandrini, C. H., van Driel-Gesztelyi, L., Thompson, B. J.,

Plunkett, S., Kovári, Z., Aulanier, G., \& Young, A. 2002, A\&A, 382, 650

Démoulin, P., Pariat, E., \& Berger, M. A. 2006, Sol. Phys., 233, 3

DeVore, C. R. 2000, ApJ, 539, 944

Evershed, J. 1910, MNRAS, 70, 217

Green, L. M., López Fuentes, M. C., Mandrini, C. H., Démoulin, P., Van Driel-

Gesztelyi, L., \& Culhane, J. L. 2002, Sol. Phys., 208, 43

Hagenaar, H. J. 1999, Ph.D. thesis, Univ. Utrecht

Kusano, K., Maeshiro, T., Yokoyama, T., \& Sakurai, T. 2002, ApJ, 577, 501

Leka, K. D., Canfield, R. C., McClymont, A. N., \& Van Driel-Gesztelyi, L. 1996, ApJ, 462, 547

Linton, M. G., Dahlburg, R. B., Fisher, G. H., \& Longcope, D. W. 1998, ApJ, 507,404
REFERENCES

Longcope, D., Beveridge, C., Qiu, J., Ravindra, B., Barnes, G., \& Dasso, S. 2007, Sol. Phys., in press, DOI: 10.1007/s11207-007-0330-7

Longcope, D. W. 1996, Sol. Phys., 169, 91

Longcope, D. W., \& Magara, T. 2004, ApJ, 608, 1106

Longcope, D. W., McKenzie, D., Cirtain, J., \& Scott, J. 2005, ApJ, 630, 596

Longcope, D. W., \& Welsch, B. T. 2000, ApJ, 545, 1089

López Fuentes, M. C., Demoulin, P., Mandrini, C. H., \& van Driel-Gesztelyi, L. 2000, ApJ, 544, 540

Low, B. C. 2006, ApJ, 649, 1064

Moffatt, H. K., \& Ricca, R. L. 1992, Proc. R. Soc. London A, 439, 411

Moon, Y.-J., Chae, J., Choe, G. S., Wang, H., Park, Y. D., Yun, H. S., Yurchyshyn, V., \& Goode, P. R. 2002, ApJ, 574, 1066

Nindos, A., Zhang, J., \& Zhang, H. 2003, ApJ, 594, 1033

November, L. J., \& Simon, G. W. 1988, ApJ, 333, 427

Pariat, E., Démoulin, P., \& Berger, M. A. 2005, A\&A, 439, 1191

Parker, E. N. 1975, ApJ, 198, 205

Pevtsov, A. A., \& Longcope, D. W. 1998, ApJ, 508, 908

Pevtsov, A. A., Maleev, V., \& Longcope, D. W. 2003, ApJ, 593, 1217

Régnier, S., Amari, T., \& Canfield, R. C. 2005, A\&A, 442, 345

Scherrer, P. H., et al. 1995, Sol. Phys., 162, 129

Schrijver, C. J., Hagenaar, H. J., \& Title, A. M. 1997, ApJ, 475, 328

St. John, C. E. 1913, ApJ, 37, 322

Tanaka, K. 1991, Sol. Phys., 136, 133

van Driel-Gesztelyi, L., Hofmann, A., Demoulin, P., Schmieder, B., \& Csepura, G. 1994, Sol. Phys., 149, 309

Welsch, B., \& Longcope, D. 2003, ApJ, 588, 620 